\title{
Photo-induced processes in heterogeneous nanosystems. From photoexcitation to interfacial chemical transformations
}

\author{
A. Emeline, ${ }^{1}$ A. Salinaro, ${ }^{1}$ V. K. Ryabchuk, ${ }^{2}$ and N. Serpone ${ }^{1, \dagger, \ddagger}$ \\ ${ }^{1}$ Department of Chemistry and Biochemistry, Concordia University, 1455 deMaisonneuve Blvd. West, \\ Montreal (Quebec), Canada H3G 1 M8 \\ ${ }^{2}$ Department of Photonics, Institute of Physics, St. Petersburg State University, \\ Ulyanovskaya 1, St. Petersburg, Russia
}

\begin{abstract}
This article briefly reviews some of our recent work carried out both from an experimental point of view as well as from a theoretical perspective to gain further understanding of the events that take place in Heterogeneous Photocatalysis. Previously, the multitude of reports from our laboratory and from many others looked at the primary photocatalytic events as involving (a) absorption of light, (b) formation of the free (electrons and holes) and/or trapped charge carriers ( $\mathrm{Ti}^{3+}$ and $\bullet \mathrm{OH}$ radicals), and (c) reaction of pre-adsorbed acceptor or donor molecules with the relevant trapped carrier. Our recent work notes that this view is reasonable if the only purpose of photocatalysis is elimination of undesirable environmental pollutants. But when we begin to query how to render a process more efficient, we need to address the primary events following photoexcitation of the photocatalyst, which in most instances has been titanium dioxide (in the anatase form). Owing to the nature of light absorption by $\mathrm{TiO}_{2}$ we resorted to examining other metal oxides, most of which are dielectric insulators with very large bandgap energies, for example zirconia $\left(\mathrm{ZrO}_{2}\right)$ and scandia $\left(\mathrm{Sc}_{2} \mathrm{O}_{3}\right)$. These dielectrics have provided added information on the photophysical events, many of which are masked by the strong light absorption in titania. Despite some of our recent progress, much remains to be done for a fuller understanding of the events that occur at the surface, which we have often considered to be the greatest and most complex defect in metal oxide particulates.
\end{abstract}

\section{INTRODUCTION}

The great interest witnessed in studies of photoinduced processes in heterogeneous systems is not surprising. What was the sphere of interest of a few research groups some years ago, we now note hundreds of such groups exploring and exploiting the potentials of heterogeneous photocatalysis. To appreciate this ongoing interest, we need only look around us to discover surfaces (on soil, buildings, walls, streets, and others) that serve as interfaces between solid, liquid and gas phases. We can see these surfaces because when exposed to sunlight they reflect and scatter the incident radiation. We also appreciate a world of colored surfaces because of selective absorption of sunlight by entities at such interfaces, the effect of which creates thermodynamically open heterogeneous systems with flow of free energy of light. Of consequence, it is relevant to query how such systems convert this free energy, what the

$\dagger$ Current address: Division of Chemistry, National Science Foundation, 4201 Wilson Blvd., Arlington VA 22230, USA.

E-mail: nserpone@nsf.gov

${ }^{\ddagger}$ Address all correspondence to this author at Concordia University.

E-mail: serpone@vax2.concordia.ca consequences of light energy conversion in heterogeneous systems are, what parameters govern and are responsible for the photostability and/or photosensitivity, and for the photoactivity of such heterogeneous systems, and what factors control photo-induced processes. These are only but a few of the questions that Nature has collectively challenged and engaged us in exploring photostimulated processes that take place in heterogeneous systems.

\section{PHOTO-INDUCED PROCESSES IN SOLIDS}

Among the countless photo-induced processes, our attention has been concentrated particularly on those photochemical processes that occur at the interface of solid/liquid and solid/gas heterogeneous systems, and on their relationship(s) with photophysical processes that take place in the solids. The first step of any photostimulated process is photoexcitation of the system resulting from absorption of photons. Typically, the solid is responsible for light absorption in a heterogeneous system. The photon energy in the near-UV/Vis spectral region corresponds to the energy of excitation of the electronic subsystem(s) of the solid. We distinguish several types of photoexcitation of solids. The first type is an intrinsic photoexcitation which leads to electron transitions from the valence band to the con- 


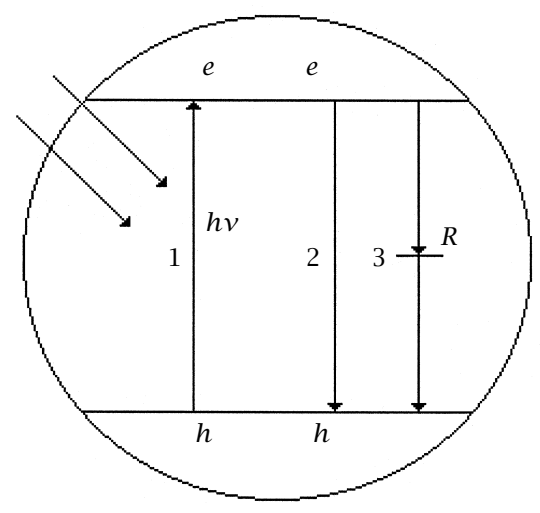

(a)

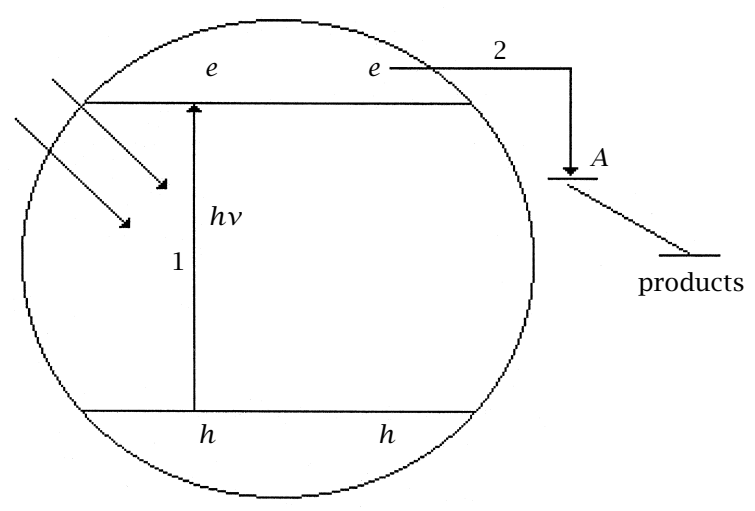

(b)

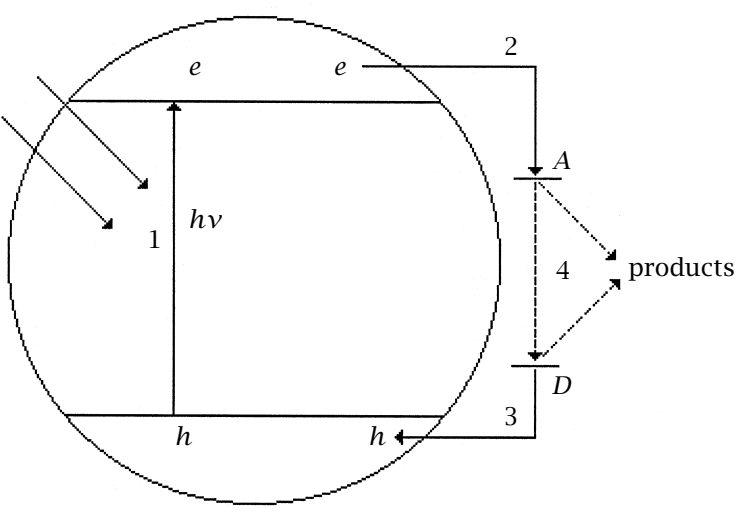

(c)

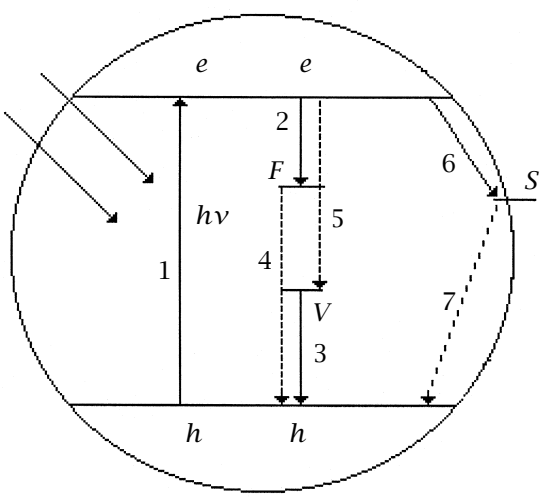

(d)

Figure 1. Schemes of excitation processes and relaxation in heterogeneous systems containing solid particles. (a) step 1: photoexcitation; step 2: complete relaxation in ideal solids (band-to-band recombination); step 3: complete relaxation in real solids (free charge carrier recombination through defect centers, R). (b) step 1: photoexcitation; step 2: interfacial electron transfer from the solid to the acceptor molecule (stoichiometric reaction, incomplete relaxation). (c) step 1: photoexcitation; step 2: interfacial transfer electron from the solid to the acceptor molecule; step 3: interfacial electron transfer from donor molecules to the solid to complete solid relaxation and the photocatalytic cycle; step 4: secondary chemical process to close the cycle of the charge carriers. (d) step 1: photoexcitation; step 2: electron trapping by defect sites to form F-type color centers (incomplete relaxation); step 3: hole trapping by defect centerss to form $V$-type color centers (incomplete relaxation); steps 4 and 5: slow recombination process through color centers to complete the relaxation of the solid; step 6: electron trapping by surface defects to form surface active (color) centers, $S$, (incomplete relaxation); step 7: deactivation of surface active centers through recombination (complete relaxation).

duction band of the solid, or to formation of excitons (bound electron-hole pairs) when the excitation energy coincides with the excitonic absorption band of that solid. This type of photoexcitation corresponds to the fundamental absorption of solids, that is band-to-band absorption.

In the ideal case of a perfect lattice structure, intrinsic absorption of light is the only possible type of electronic photoexcitation of solids. The opposite processes of excitation decay in an ideal solid, which return the solid to its initial ground state, are the band-to-band electron-hole recombination or exciton decay (Figure 1a). When the solid is an integral component of a heterogeneous system, interfacial transfer of charge carriers becomes possible. This is synonymous with a well-known three-level photochemical system (Figure 1b). The pathway corresponds to a simple stoichiometric heterogeneous photoreaction. However, if the energy diagram of Figure $1 \mathrm{~b}$ were expanded to a four-level system (see Figure 1c), when the charge carrier cycle is closed due to redox reactions with both acceptor and donor molecules at the surface, which leads 
to complete relaxation of the solid back the ground state, the process can then be viewed as being photocatalytic. Thus, any stoichiometric surface photoreaction can be considered as an incomplete photocatalytic cycle. The result of such an incomplete cycle is that at the end of the reaction the system goes into a new, higher energy state. An example of such a process is the photo-induced adsorption of molecules on metaloxide surfaces.

\section{PHOTOCHEMICAL PROCESSES ON SOLIDS}

There are two well-known surface stoichiometric photochemical reactions that can be identified immediately: (i) the photostimulated adsorption of dioxygen (acceptor molecules), and (ii) the photostimulated adsorption of dihydrogen (donor molecules), both of which result in a new state of the heterogeneous system with charged species adsorbed on the solid's surface. If these two processes occurred simultaneouslythey would yield a reaction identifiable as the photocatalyzed oxidation of hydrogen to water. Nonetheless, such a simple mechanism gives but just a small reflection of the real processes that take place on solids and at interfaces of heterogeneous systems.

\section{NATURE OF SOLIDS}

It is important to recognize that no solid is perfect and that none possesses an ideal lattice structure. Even for an ideal solid, its surface interrupts the translation symmetry of the lattice and consequently disturbs the periodic potential of the solid, and leads to the creation of so-called regular surface states. These surface states have different energy distributions compared to the distribution of defects in the bulk. Consequently, they will display their own absorption bands causing specific surface absorption with the energy of the photons absorbed being typically smaller than in the fundamental absorption (Figure 1d). The result of surface absorption of light is the formation of surface free electrons and holes, or surface excitons, which can participate in redox surface reactions just as much as the charge carriers generated in the bulk do subsequent to their migration to the surface. Surface reconstruction and surface relaxation processes may lead to changes in the energy distribution of carriers, and may even lead to formation of a new structure of the solid in the subsurface space. Adsorption of molecules on the surface also changes the charge-carrier and energy distribution at the surface. All these events result in a new charge and energy distribution in subsurface space (i.e., the space-charge layer), which typically manifests itself as band-bending in semiconductors.

Even with the most advanced methods of crystal growth, solids still contain lattice defects at concen- trations around $10^{13}-10^{14} \mathrm{~cm}^{-3}$, whereas the typical concentrations of defects and impurities in commercial samples fluctuate around $10^{18} \mathrm{~cm}^{-3}$. Note that this concentration is usually much greater than the concentration of photogenerated free charge carriers in the solids under moderate photoexcitation. Consequently, one must expect the defects to play an important role in photoexcitation and relaxation processes in heterogeneous systems. In fact, the defects create a local distortion of the periodic potential in the solid's lattice. This results in the appearance of local energy levels within the band gap that lead to changes in the optical and electronic properties of solids.

Defects often play a dual role during photoexcitation of solids (Figure 2). First, their presence, together with surface states, causes extrinsic absorption of light at energies much lower than the bandgap of solids [1, 2]. Depending on the type of defects and on the mechanism of their photoexcitation, extrinsic absorption also leads to photoinduced generation of both electrons and holes [1-4]. Second, lattice defects can serve as carrier traps in processes of charge carrier recombination and in the formation of photoinduced defects (color centers). Carrier decay occurring through lattice defects is a more efficient process than band-to-band recombination since there is no limitation regarding charge carrier momentum conservation. As well, the system's energy conservation rule is more easily satisfied. Results of carrier trapping by pre-existing defects is the formation of new photo-induced defect states, or generation of a new type of defects which lead to a new energy distribution of charge carriers in solids.

\section{PHOTOPHYSICAL EVENTS IN SOLIDS}

Photogenerated color centers differ from each other by the energy difference $E_{d}$ between the level of the trapped carrier within the energy bandgap of the semiconductor (or of the insulator) and the bottom of the conduction band for electron centers, and the top of the valence band for hole centers (see Figure 2 for details). From this point of view, a given photogenerated center can be described as a shallow center (or trap) if the energy of the trapped carrier is comparable to the energy of thermal excitation of the crystal lattice (i.e., if $E_{d} \sim k T$ ), or as a deep energy center (trap) if $E_{d} \gg k T$. The difference between the various types of photogenerated centers is determined by the dominant decay pathway that brings the solid back to the initial state (without trapped carrier). In general, any photo-induced center can lose a carrier as a result of: (i) thermal ionization with a thermal quenching probability given by $q_{\text {th }}$; (ii) recombination with a charge carrier of the opposite sign for which the recombination probability is $q_{\text {rec }}$; (iii) photoionization caused by incident photons, $q_{\mathrm{ph}}$; and (iv) tunnelling recombination of adjacent hole 


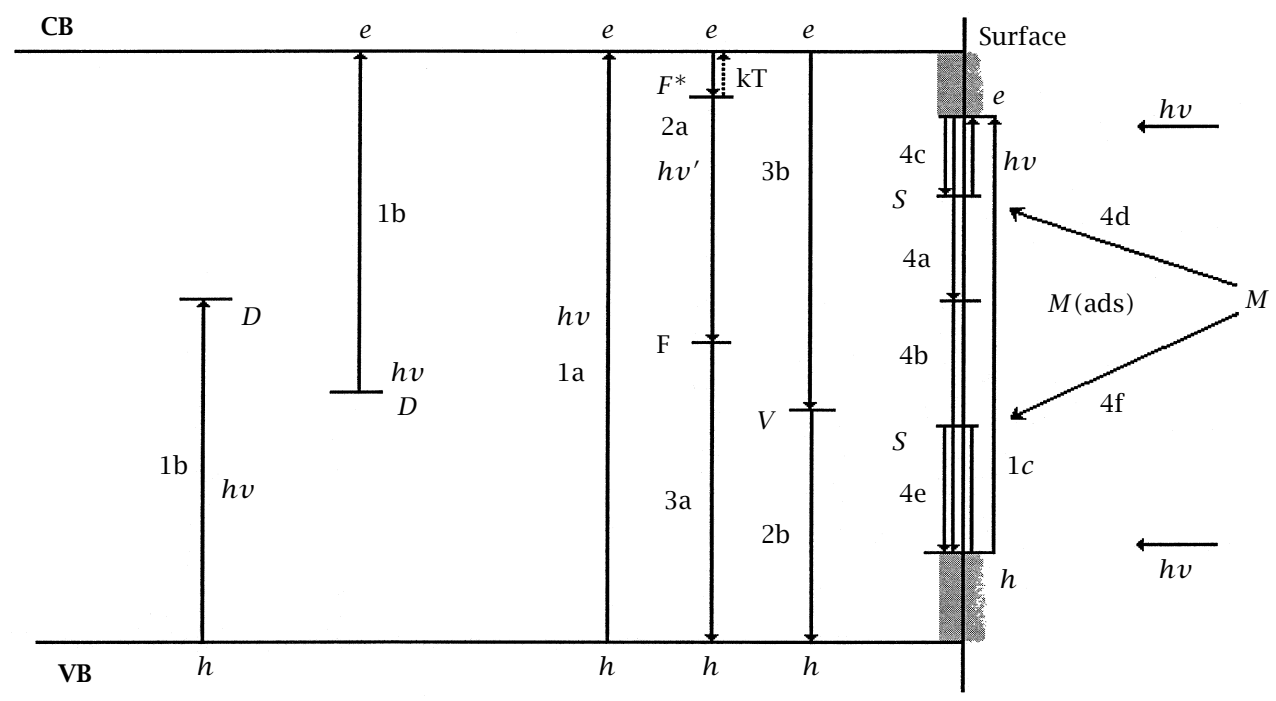

Figure 2. Summary of photoexcitation and relaxation processes in solids as part of heterogeneous system: step 1a: intrinsic (band-to-band) photoexcitation corresponding to the fundamental absorption of the solid; step 1b: extrinsic photoexcitiaiton of bulk defects $\left(D_{b}\right)$ in solids leading to generation of free carriers; step 1c: photoexcitation of intrinsic and extrinsic surface states generating surface charge carriers; steps $2 a$ and $2 b$ : charge carrier trapping by bulk defects to form color centers; steps $3 a$ and $3 b$ : recombination of free charge carriers with carriers trapped by color centers; steps $4 a$ and $4 c$ : free charge carrier trapping by deep and shallow surface traps (surface defects) to form surface active centers; step 4 b: recombination between free and trapped charge carriers by surface active centers; step 4e: thermoactivated deactivation of surface active centers; steps $4 d$ and 4f: chemical interaction of molecules with electron and hole surface active centers.

and electron centers, $q_{\text {tun }}$. The total probability of decay of these centers is then given by,

$$
q=q_{\mathrm{th}}+q_{\mathrm{rec}}+q_{\mathrm{ph}}+q_{\mathrm{tun}}
$$

Following the work of Siline and Trukchin [5] for the simplest case of centers with one trapped carrier, the kinetics of their formation typically follow an exponential growth and approach the steady-state concentration of photo-induced defects $N_{d}$ as given by,

$$
N_{d}=N_{o}\left(\frac{p}{p+q}\right)
$$

where $N_{o}$ is the number of pre-existing defects of a given type, $p$ is the probability of photocarrier trapping defined, as are the corresponding quenching probabilities $q$, as the number of events per unit time. For shallow traps, thermal ionization usually dominates, that is $q_{\text {th }} \gg q_{\text {rec }}+q_{\text {ph }}+q_{\text {tun }}$. In contrast, thermal ionization can be neglected for deep energy centers, i.e. $q_{\text {th }} \ll q_{\text {rec }}+q_{\text {ph }}+q_{\text {tun }}$. In the latter case, centers that have a high capability to capture a carrier of the opposite sign, i.e., for $q_{\text {rec }} \gg p$, can serve as effective recombination centers. These centers do not accumulate in considerable quantity in illuminated crystals. At the same time, recombination centers are mainly responsible for the concentrations of photogenerated carriers in wide bandgap solids because the direct band-to-band recombination is rather inefficient compared with the one involving deep energy centers. Contrasting recombination centers, deep energy centers, for which $q_{\mathrm{rec}} \sim$ $p$, do accumulate in sufficient amount in solids under illumination. Such defects are referred to as color centers because they usually have absorption bands placed in the UV and visible regions of the spectrum and determine the lasting stable color of pre-illuminated, initially colorless wide bandgap solids (see Figures $1 \mathrm{~d}$ and 2). Photoionization of color centers by the actinic light may, to some extent, reduce the color saturation level under illumination when $q_{\text {rec }} \sim q_{\mathrm{ph}}$. An increase in temperature causes an increase of the probability $q_{\mathrm{th}}$ for the thermal ionization of color centers, which de facto may become the dominant pathway for the decay of color centers.

In our recent studies we have examined the processes of formation and decay of photoinduced centers under steady-state photoexcitation conditions, as well as under picosecond laser pulse excitation [1-4, 6, 7]. The spectra of color centers induced in some of the solids examined under steady-state irradiation are presented in Figures 3a and $3 \mathrm{~b}$ for irradiation of $\mathrm{ZrO}_{2}$ and $\mathrm{Sc}_{2} \mathrm{O}_{3}$, respectively, in vacuo, in oxygen and in hydrogen. Figure 3c illustrates the temporal evolution of photo-induced color centers in colloidal titania, $\mathrm{TiO}_{2}$, following picosecond laser pulse excitation. These centers are formed as a result of carrier/exciton trapping by pre-existing defects (anion $V_{a}$ and cation $V_{c}$ vacan- 

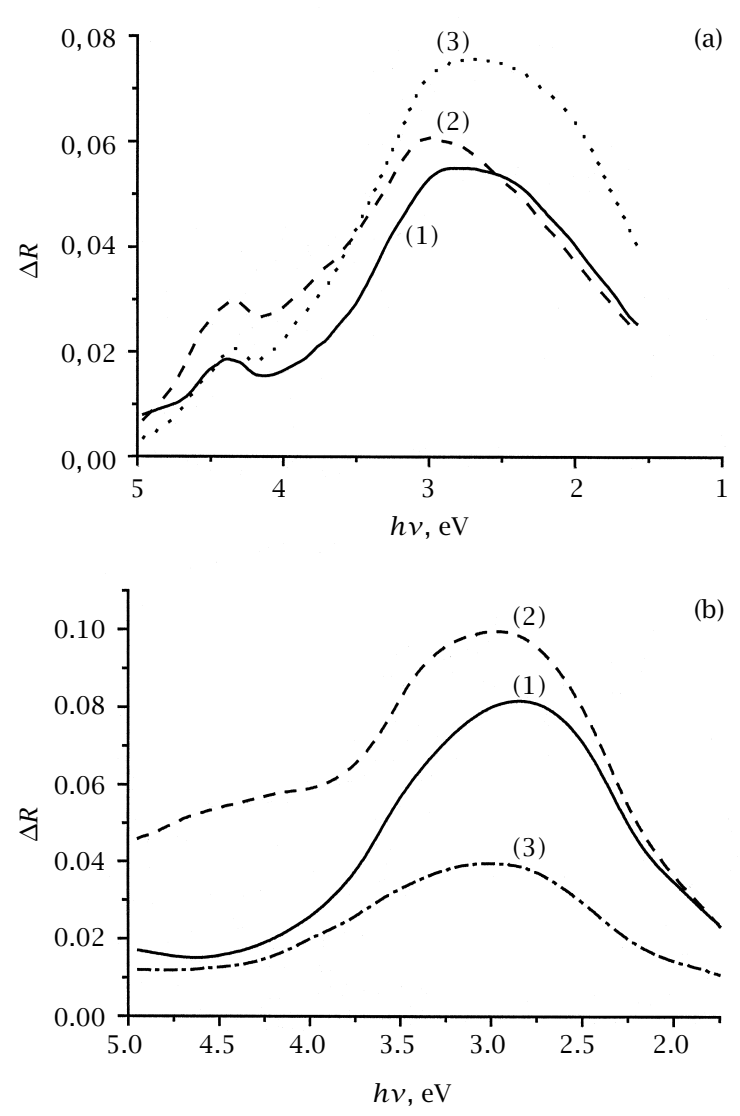

(c)

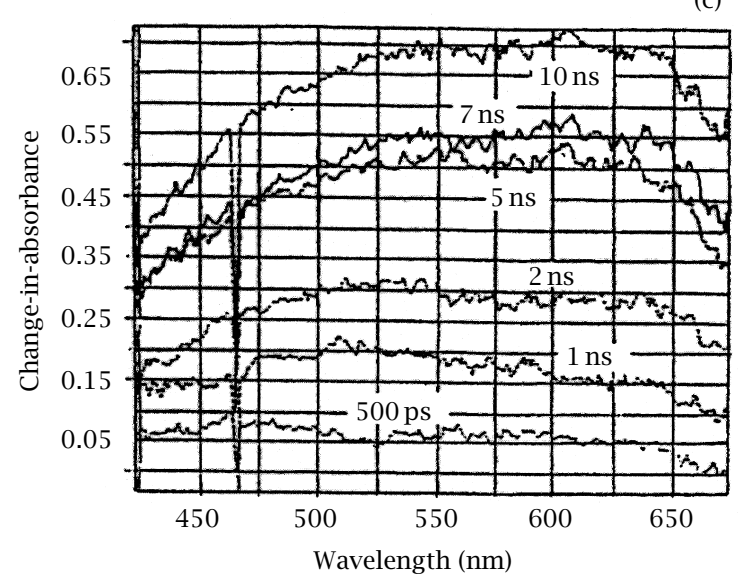

Figure 3. (a) Absorption spectra of photoinduced color centers in powdered $\mathrm{ZrO}_{2}$ particles formed under irradiation in vacuo (1), in the presence of dioxygen (2), and in the presence of dihydrogen (3). From reference [1] \{Copyright by the American Chemical Society?. (b) Absorption spectra of photoinduced color centers in powdered $\mathrm{Sc}_{2} \mathrm{O}_{3}$ particles formed under irradiation in vacuo (1), in the presence of dioxygen (2), and in the presence of dihydrogen (3). From reference [2] \{Copyright by the American Chemical Society\}. (c) Absorption spectra of photoinduced color centers in colloidal $\mathrm{TiO}_{2}$ particles formed after picosecond laser pulse excitation. From reference [7] \{Copyright by the American Chemical Society?. cies), and by some ions in irregular (defect) positions in the lattice, for example $\mathrm{Ti}^{4+}{ }_{\text {irreg }}$ in titania (or $\mathrm{Zr}_{\text {irreg }}^{4+}$ in zirconia).

$$
\begin{aligned}
& \mathrm{V}_{\mathrm{a}}+\mathrm{e} \longrightarrow F, \\
& \mathrm{~V}_{\mathrm{a}}+\mathrm{e}^{\mathrm{O}} \longrightarrow F+\mathrm{h}, \\
& \mathrm{V}_{\mathrm{c}}+h \longrightarrow V, \\
& \mathrm{~V}_{\mathrm{c}}+\mathrm{e}^{\mathrm{O}} \longrightarrow V+e, \\
& \mathrm{Ti}^{4+}{ }_{\text {irreg }}+\mathrm{e} \longrightarrow \mathrm{Ti}^{3+}{ }_{\text {irreg }}, \\
& \mathrm{Ti}^{4+}{ }_{\text {irreg }}+\mathrm{e}^{\mathrm{O}} \longrightarrow \mathrm{Ti}^{3+}{ }_{\text {irreg }}+\mathrm{h} .
\end{aligned}
$$

The kinetics of formation of color centers under steadystate irradiation are illustrated in Figure $4 \mathrm{a}$ for $\mathrm{ZrO}_{2}$ and Figure $4 \mathrm{~b}$ for $\mathrm{Sc}_{2} \mathrm{O}_{3}$ systems. It is clear that after a period of growth, the concentration of the color centers reaches steady-state, which is determined by the competing processes of formation and decay of the color centers from recombination events (see stages (4a)-(4f)) as determined by equation (1).

$$
\begin{gathered}
F+\mathrm{h} \longrightarrow \mathrm{V}_{\mathrm{a}}, \\
V+\mathrm{e} \longrightarrow \mathrm{V}_{\mathrm{c}}, \\
V+\mathrm{e}^{\mathrm{o}} \longrightarrow \mathrm{V}_{\mathrm{c}}+\mathrm{h}, \\
F+\mathrm{e}^{\mathrm{o}} \longrightarrow \mathrm{V}_{\mathrm{a}}+\mathrm{e}, \\
\mathrm{Ti}^{3+}{ }_{\text {irreg }}+\mathrm{h} \longrightarrow \mathrm{Ti}^{4+}{ }_{\text {irreg }}, \\
\mathrm{Ti}^{3+}{ }_{\text {irreg }}+\mathrm{e}^{\mathrm{o}} \longrightarrow \mathrm{Ti}^{4+}{ }_{\text {irreg }}+\mathrm{e} .
\end{gathered}
$$

The same processes (stages (3) and 4) occur after the solid is photoexcited by picosecond laser pulses. The time evolution of photoinduced electron centers, $\mathrm{Ti}^{3+}$, in $\mathrm{TiO}_{2}$ is presented in Figure 4c. The difference in for pulse-induced and stationary-excited processes the formation of color centers lies in the different probabilities of formation and decay of the centers, which dictates the different time evolution and different time scales of the relevant processes. First, a disparity in the excitation intensity causes the concentration of photogenerated charge carriers to be different. For stationary irradiation at moderate light intensity, the carrier concentration is much smaller than the concentration of defects, whereas under laser pulse excitation the concentrations of charge carriers and defects may be comparable. Second, during steady-state irradiation $F$ and $V$ centers (see stages (3a)-3c) will form such that the steady-state irradiation event will also activate these centers, contrary to the case of laser pulse excitation where irradiation will only encounter the original pre-existing defects on the metal oxide particles. For example, in the original state before laser pulse excitation, there exist only anion and cation vacancies, and consequently only processes of charge carrier trapping by these vacancies is possible. This means that any faster process (either electron or hole trapping) occurs first with a probability dictated by the trapping crosssection of the corresponding type of vacancy and by the 

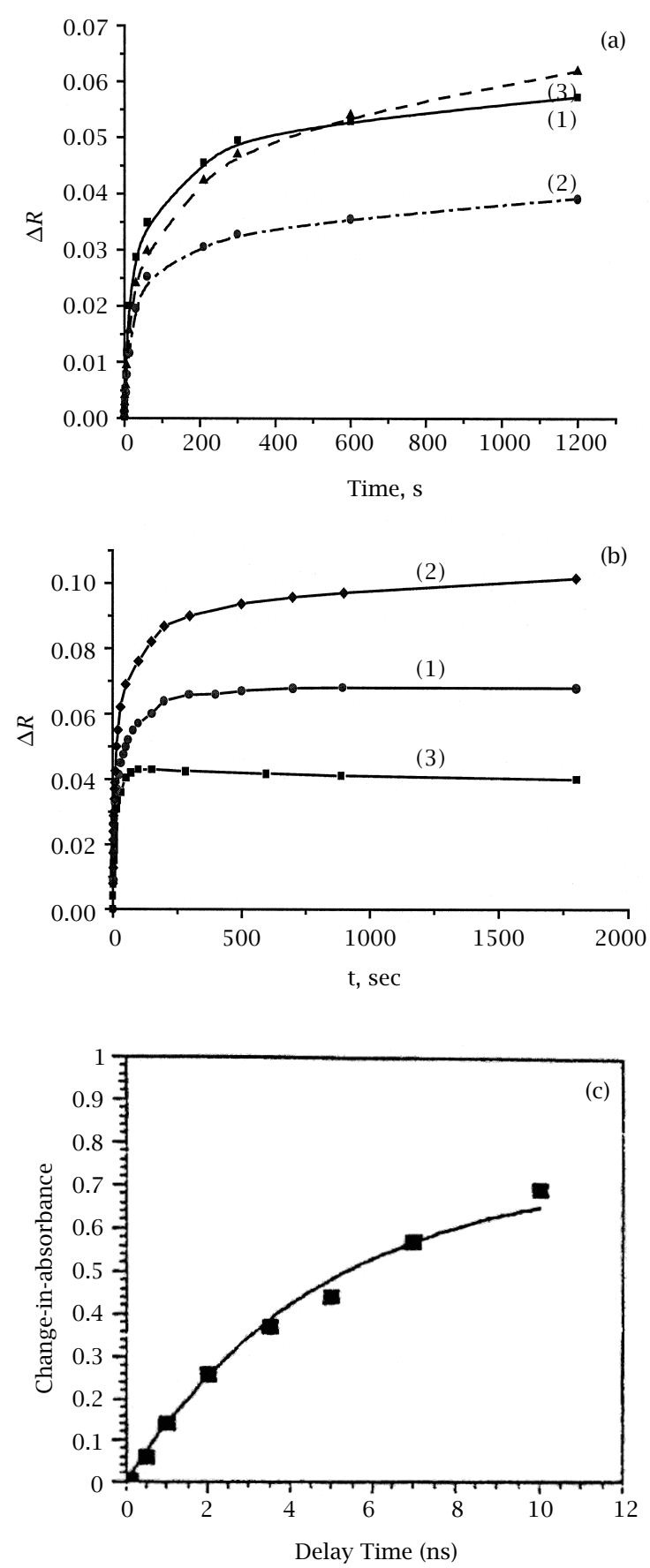

Figure 4. (a) Kinetics of formation of F-type color centers in powdered $\mathrm{ZrO}_{2}$ particles under stationary photoexcitation in vacuo (1), in the presence of dioxygen (2), and in the presence of dihydrogen (3). (b) Kinetics of formation of $V$-type color centers in powdered $\mathrm{Sc}_{2} \mathrm{O}_{3}$ particles under stationary photoexcitation in vacuo (1), in the presence of dioxygen (2), and in the presence of dihydrogen (3). From reference [2] \{Copyright by the American Chemical Society\}. (c) Kinetics of fromation of $\mathrm{Ti}^{3+}$-type color centers in colloidal $\mathrm{TiO}_{2}$ after picosecond laser pulse excitation. From reference [7] \{Copyright by the American Chemical Society\}. mobility of the corresponding type of carriers, followed by slower processes of trapping and recombination of charge carries of the opposite sign. By contrast, under stationary irradiation all these processes may occur simultaneously since some photogenerated color centers will have been formed during irradiation. Consequently, lifetimes of charge carriers and photo-induced centers assessed by time-resolved laser techniques will not correspond to those determined under stationary irradiation conditions.

The rates of recombination of free holes with $F$ centers (trapped electrons; stage (4a)) and free electrons with $V$ centers (trapped holes; stage (4b)) are slow compared to those occurring at recombination centers $R$. Ordinarily, $R$ centers are also deep energy carrier traps that can later capture carriers of both signs. These processes can also be expressed by quasi-chemical reactions (not shown) similar to stages (3a), (3b) and (4a), (4b). The distinction between color centers and recombination centers is the trapping efficiency of the second carrier. Color centers (small rate constants for stages (4a) and (4b), or small cross sections of second carrier trapping) accumulate in dielectrics in amounts comparable to those for primary defects (in our notation, $V_{a}$ and $V_{c}$ ). By contrast, the ultimate concentrations of recombination centers $R^{-}$and $R^{+}$(in the charge states corresponding to $F$ and $V$ centers, respectively) are much smaller than the concentration of $R$ because of the large cross sections in the trapping of the second carrier. In this description, intrinsic defects as well as impurities can serve as primary defect species for both color and recombination centers, depending on the peculiarity(ies) of their interaction with free carriers. In other words, the process of coloration of solids may be taken as an incomplete recombination through the defects when trapping of the second carrier is not fulfilled. Note the analogy between coloration (as incomplete recombination) and recombination on the one hand, and surface stoichiometric photochemical reaction and photocatalysis on the other (see for example, Figures 1 and 2). The difference between these two pairs of processes is that the photocatalytic process closes the external charge carrier cycle, whereas the recombination process results in the complete internal chargecarrier cycle. To support this analogy, we suppose that photoprocesses of defect formation can also be considered as a reduction or oxidation of intrinsic or extrinsic pre-existing defect sites. Indeed, electron trapping by some defects (e.g., by $\mathrm{Ti}^{4+}$ to form $\mathrm{Ti}^{3+}$ ) can be designated as a reduction process, whereas hole trapping to produce such hole centers as the $\mathrm{O}^{-\bullet}$ radical anion state localized at a negatively charged defect in a metal oxide lattice can be described as photooxidation of oxide $\mathrm{O}^{2-}$ anions. Also, according to the electronic theory of catalysis [8], any adsorbed molecule is a defect at the solid's surface and can act as a carrier trap just as much as intrinsic lattice defects do in the bulk of the solid. 
Adsorbed species such as, for example, the localized $\mathrm{O}_{2}{ }^{-\bullet}$ anion radical formed through electron trapping by molecular oxygen is one such defect. In terms of defect formation, the interaction of $\mathrm{O}_{2}^{-\bullet}$ anion radicals with photo-holes at the $\mathrm{TiO}_{2}$ particle surface, which leads to photodesorption of $\mathrm{O}_{2}$ molecules $[9,10]$ or to dissociation of the $\mathrm{O}_{2}^{-}$anion radical into oxygen atoms, as proposed by Formenti and Teichner [11], can be characterized as recombination decay of photo-induced centers. Clearly, these external and internal cycles compete with each other. We then infer that this is competition between physical (internal cycle) and chemical (external cycle) pathways for the system to relax after photoexcitation.

Irradiation of solids in vacuo leads to formation of photoinduced color centers (see Figures 3 and 4). Those photo-induced centers formed at the surface can serve as reactive centers for surface chemical processes: $V$ type holes centers $\left(\mathrm{O}_{\mathrm{s}}{ }^{-\bullet}\right)$ serve as reactive centers for oxidative reactions with donor molecules, whereas electron $F$-type centers or low-coordinated metal ions with trapped electrons $\left(\mathrm{Ti}^{3+}, \mathrm{Zr}^{3+}\right)$ lead to a reductive reaction with acceptor molecules.

Absorption changes caused by adsorption of donor $\left(\mathrm{H}_{2}\right)$ and acceptor $\left(\mathrm{O}_{2}\right)$ molecules on pre-irradiated samples corresponding to the absorption spectra of surface active (color) centers are presented in Figure 5. These centers are long-lived centers because as color centers they belong to the class of deep traps, and consequently the probability of their thermoionization is very low, that is $q_{\mathrm{th}} \longrightarrow 0$. After irradiation is terminated, the probability of photoionization $q_{\mathrm{ph}}$ is 0 and the probability of recombination decay $q_{\text {rec }}$ is also 0 since there are no free charge carriers left in the solid. Thus, the total probability of decay of centers is 0 (equation (1)). These centers are responsible for the "memory" effect when pre-irradiation of solids causes consequent dark reactions. However, under irradiation the recombination probability for the decay of color centers on the surface and in the bulk is not 0 , that is $q_{\text {rec }} \neq 0$. Note that if solids are excited in their fundamental absorption band, the probability of photoionization of active (color) centers may be negligible compared to the recombination probability, that is $q_{\mathrm{ph}} \ll q_{\mathrm{rec}}$. Thus, under irradiation the active states of surface defects have a limited lifetime to initiate surface chemical reactions.

To summarize what has been noted above we propose the following series of reactions of surface photochemical processes:

$$
S+\mathrm{e} \rightarrow \mathrm{S}^{-} \quad\left(k_{c}\right)
$$

(formation of active surface center),

$$
S^{-}+\mathrm{h} \rightarrow \mathrm{S} \quad\left(k_{R}\right)
$$

(recombination decay of active center),
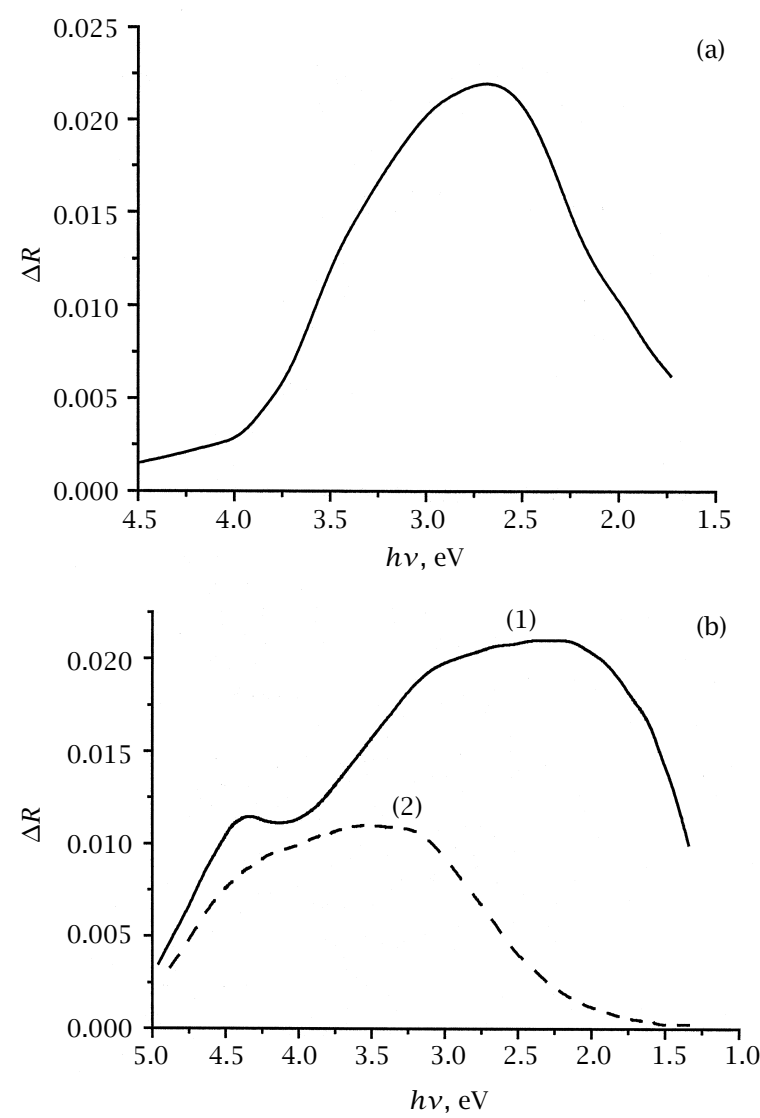

Figure 5. (a) Absorption spectrum of long-lived surface hole active centers on powdered $\mathrm{Sc}_{2} \mathrm{O}_{3}$ particles. From reference [2] \{Copyright by the American Chemical Society\}. (b) Absorption spectrum of long-lived surface electron (1) and hole (2) active centers on powdered $\mathrm{ZrO}_{2}$ particles. From reference [1] \{Copyright by the American Chemical Society\}.

$$
\begin{aligned}
S^{-}+M_{\text {(gas) }} \longrightarrow & M^{-} \quad\left(k_{a}\right) \\
& \text { (reaction with molecule). }
\end{aligned}
$$

Formation of surface active (color) centers occurs via capture of free electrons and holes by the surface traps S. For completeness, creation of electron active centers $\mathrm{S}^{-}$are indicated in stage $(5 \mathrm{a})\left(k_{c}\right)$. The centers interact with molecules $M$ to yield the intermediate species $M^{-}$ (stage (5c), $k_{a}$ ). Stage (5b) describes the recombination pathway for the decay of surface active centers.

In the quasi-stationary approximation $\left(d\left[S^{-}\right] / d t=\right.$ $0 ; d[\mathrm{e}] / d t=0$; and $d[\mathrm{~h}] / d t=0$ ), the rate of the photochemical reaction $d[M] / d t$ can be expressed in terms of steady-state surface concentrations of photoelectrons [e] and photo-holes [h], namely

$$
-\frac{d[m]}{d t}=\frac{k_{c} S[\mathrm{e}] k_{a}[M]}{\left(k_{R}[\mathrm{~h}]+k_{a}[M]\right)} .
$$


We now consider further a more detailed expression for the surface concentration of charge carriers. We assume that the steady-state concentration of photoelectrons and photo-holes is given by equations (7) and (8):

$$
\begin{aligned}
{[e]=\alpha \rho \tau_{e} } & =g \tau_{e}, \\
{[h]=\alpha \rho \tau_{h} } & =g \tau_{h},
\end{aligned}
$$

where $g$ denotes the volume rate $\left(\mathrm{cm}^{-3} \mathrm{~s}^{-1}\right)$ of generation of charge carriers and $\alpha$ is the absorption coefficient $\left(\mathrm{cm}^{-1}\right)$. Consequently, taking into account equations (7) and (8), equation (6) can be transformed to the form that summarizes the dependence of the reaction rate on the concentration of reagent and on the photon flow $(\rho)$ :

$$
\left(\frac{d[M]}{d t}\right)(\rho,[M])=\frac{\alpha \rho[M]}{(\beta \rho+\gamma[M])},
$$

where the coefficients $\alpha, \beta$, and $\gamma$ are independent of photon flow $\rho$ and reagent concentration [M]. Equation (9) completely describes the experimental dependencies of the rate of the photodegradation of phenol in $\mathrm{TiO}_{2}$ dispersions on both photon flow and phenol concentration [12], and the dependencies of the rate of photostimulated adsorption of dioxygen on $\mathrm{ZrO}_{2}$ particles on both oxygen pressure and photon flow [13]; they are presented in Figure 6. Note that equation (9) can be cast into the Langmuir-Hinshelwood form when the photon flow $\rho$ is constant. Also note that equation (9) can be used to describe all the experimental dependencies of the reaction rate on light intensity (photon flow). In fact, when $\beta \rho \ll \gamma[M]$ equation (9) gives the linear dependence on light intensity observed in some recent studies by Emeline and coworkers [13] and by Basov et al. [14], whereas when $\beta \rho \gg \gamma[M]$ the reaction rate is independent of photon flow as reported by Ollis [15]. In other cases, when $\beta \rho$ is comparable to $\gamma[M]$ the dependence of the reaction rate on photon flow is sub-linear; at certain ratios between these two terms the dependence may be similar to the square-root dependence reported by Emeline and coworkers [12, 13]. Thus, rate dependencies on light intensity and on concentration of reagent are in fact interdependent. The reason for such behavior is the competition between the physical pathway (internal cycle, Figure 1d) of system excitation decay through recombination involving surface active (color) centers and the chemical pathway (external cycle, Figures $1 \mathrm{~b}$ and 1c) through chemical interaction between molecules and surface active centers. Indeed, at sufficiently high concentrations $[M]$, or at low light intensity (that is, for $\beta \rho \ll \gamma[M]$ ) recombination

\section{DEPOSITION OF SILVER (CLUSTERS) $\mathrm{ON} \mathrm{TIO}_{2}$ PARTICLES}

Our conclusion of competition between the physical
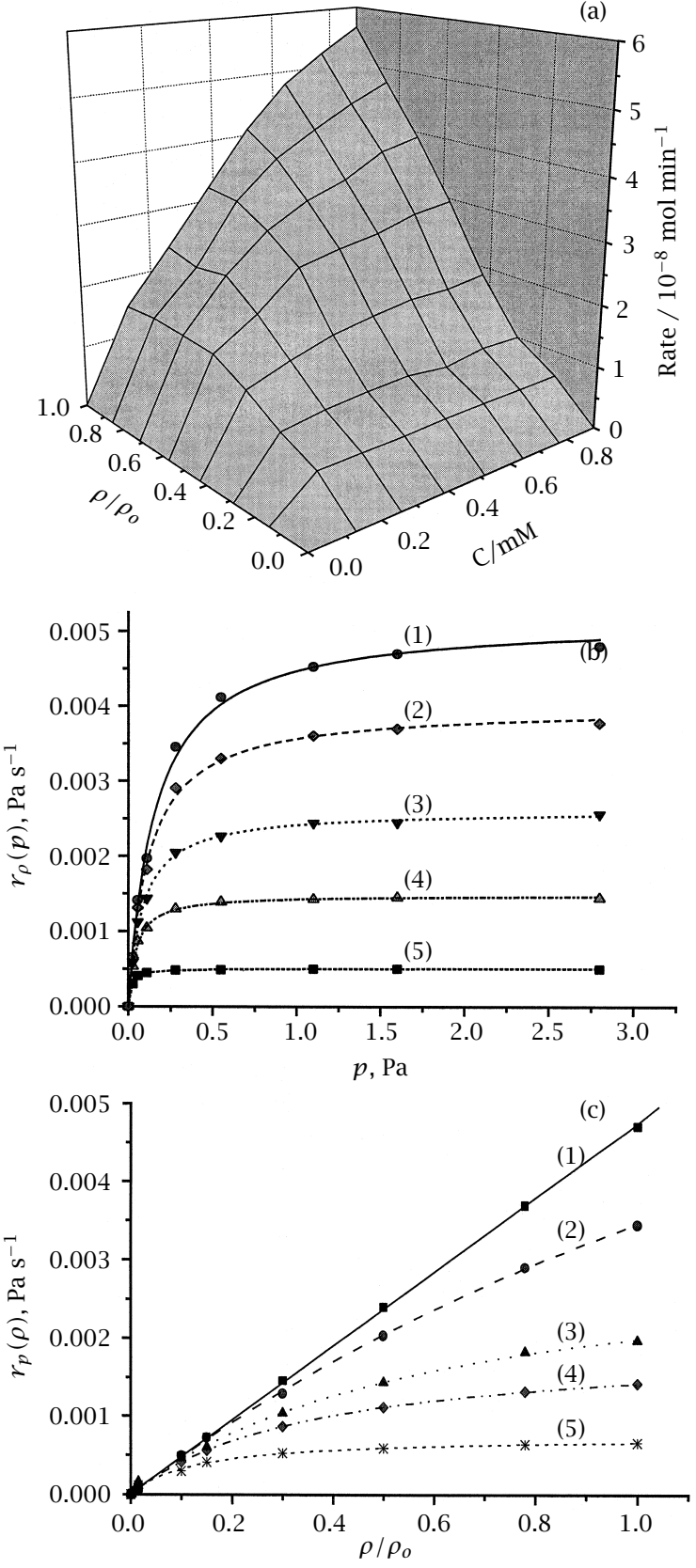

Figure 6. (a) Three-dimensional dependence of the rate of phenol photodegradation in illuminated aqueous $\mathrm{TiO}_{2}$ dispersions on the concentration of phenol and on the photon flow of the actinic light. (b) Dependencies of initial rates of oxygen photo-adsorption, $r_{\rho}(p)$, on pressure $p$ at different photon flow $\left(\rho_{o}=1 \times 10^{15}\right.$ photons $\left.\mathrm{cm}^{-2} \mathrm{sec}^{-1}\right):(1) \rho=\rho_{o}$; (2) $\rho=0.78 \rho_{o}$; (3) $\rho=0.5 \rho_{o}$; (4) $\rho=0.3 \rho_{o}$; (5) $\rho=0.1 \rho_{o}$. From reference [13] \{Copyright by the American Chemical Society\}. (c) Dependencies of initial rates of oxygen photoadsorption as $r_{p}(\rho)$ on photon flow $\rho\left\{\right.$ as $\left.\rho / \rho_{o}\right\}$ at different initial oxygen pressures: (1) $p=2.8 \mathrm{~Pa}$; (2) $p=0.28 \mathrm{~Pa}$; (3) $p=0.11 \mathrm{~Pa}$; (4) $p=0.028 \mathrm{~Pa}$; (5) $p=0.028 \mathrm{~Pa}$. The straight line corresponds to the first-order process dependence on photon flow. From reference [13] \{Copyright by the American Chemical Society?. 
recombination pathway through surface active centers and surface chemical reaction was supported by the time-resolved spectroscopic data for the photocatalytic deposition of silver on nanoparticulate $\mathrm{TiO}_{2}$ in a solid/liquid heterogeneous system consisting of $\mathrm{TiO}_{2}$ particles and silver acetate as reported by Sahyun and Serpone [7]. The system was excited by picosecond laser pulses in the presence of high concentrations of alcohol pre-adsorbed on the particle surface. The alcohol served as an effective hole scavenger to prevent recombination decay of photoinduced surface active (color) centers $\mathrm{Ti}^{3+}$ (stage (4e)). As a result, the dependencies of the concentration of photoinduced surface active centers $\mathrm{Ti}_{\mathrm{s}}{ }^{3+}$ and of the concentration of reaction product $\mathrm{Ag}^{\mathrm{O}}$ that formed during the reductive interactions with surface active sites (reaction (10)) on the energy of the laser pulse was linear (Figure 7), whereas the extent

$$
\mathrm{Ag}^{+}+\mathrm{Ti}_{\mathrm{s}}{ }^{3+} \longrightarrow \mathrm{Ag}^{\mathrm{o}}+\mathrm{Ti}_{\mathrm{s}}{ }^{4+}
$$

of absorption by $\mathrm{Ag}^{\mathrm{O}}$ was nearly independent of concentration of $\mathrm{Ag}^{+}$in solution. This infers that because of effective hole scavenging by the alcohol, the recombination of holes with $\mathrm{Ti}^{3+}$ (stage (4e)) was shut down and the lifetime of surface active centers was then long enough to permit the centers to react with any amount of $\mathrm{Ag}^{+}$used in our experiments $\left[\mathrm{Ag}^{+}\right] \geq$ $5 \times 10^{-5} \mathrm{M}$. This assumption is strongly supported by the kinetics of formation of $\mathrm{Ti}^{3+}$ species (see Figure 4c) where no decay through recombination is observed.

\section{CHARGE CONSERVATION IN SOLIDS}

The charge conservation law requires that the concentration of electrons and holes trapped in color centers during irradiation in vacuo under steady-state conditions (since the concentration of free charge carriers can be neglected compared to that of trapped carriers) or after irradiation must be equal. That is,

$$
F_{o}=V_{o}
$$

Then, at high concentration of donor molecules (D), when recombination through surface hole centers is shut down, the charge conservation law requires that

$$
F_{\mathrm{D}}=V_{\mathrm{D}}+\mathrm{D}^{+}
$$

where $\mathrm{D}^{+}$is the concentration of cation radicals formed by donor molecules with trapped holes, and $F_{\mathrm{D}}$ and $V_{\mathrm{D}}$ are the concentrations of electron and hole color centers, respectively, in the solid in the presence of donor molecules at the interface. At high concentrations of acceptor molecules (A), expression (11) transforms into

$$
F_{\mathrm{A}}+\mathrm{A}^{-}=V_{\mathrm{A}}
$$

where $\mathrm{A}^{-}$is the concentration of anion radicals formed
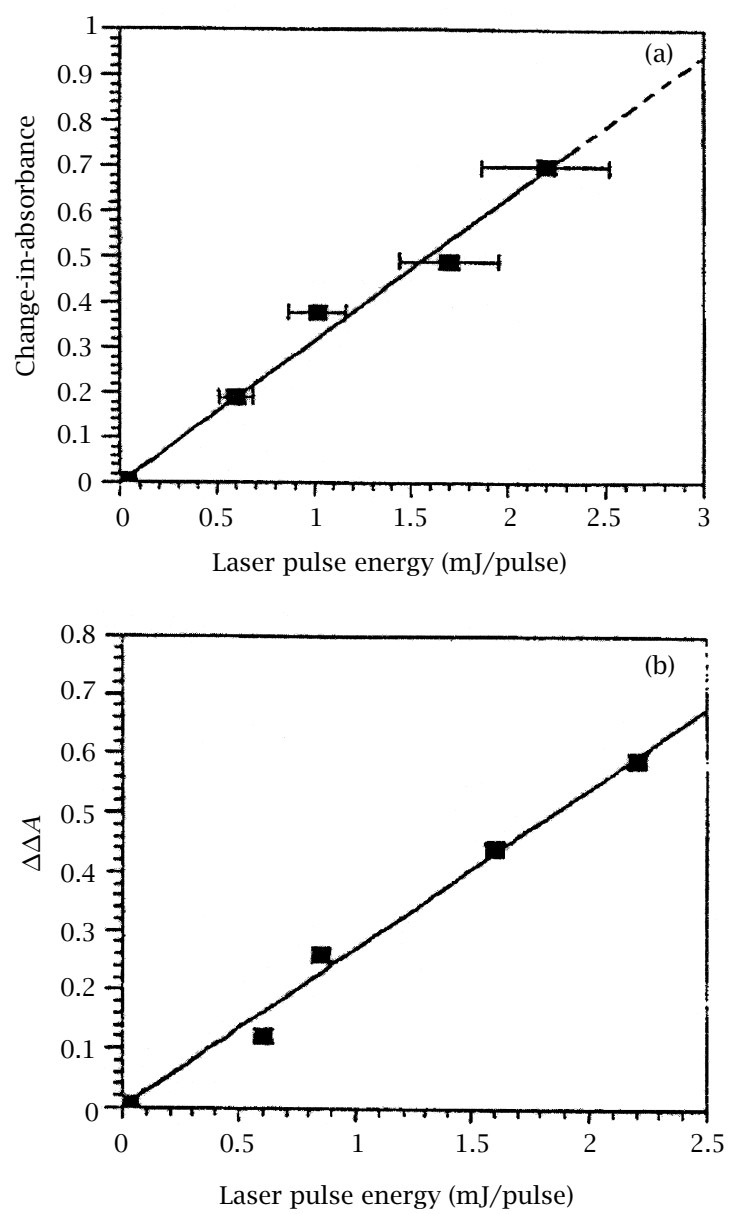

Figure 7. (a) Dependence of the amount of the laser pulseinduced surface active centers $\mathrm{Ti}_{\mathrm{s}}{ }^{3+}$ on the laser pulse energy. From reference [7] \{Copyright by the American Chemical Society\}. (b) Dependence of the amount of photoreduced silver $\mathrm{Ag}^{\circ}$ on the laser pulse energy. From reference [7] \{Copyright by the American Chemical Society\}.

by acceptor molecules with trapped electrons, and $F_{\mathrm{A}}$ and $V_{\mathrm{A}}$ are the concentrations of electron and hole color centers, respectively, in the solid in the presence of acceptor molecules at the interface. For the general case of a complete photocatalytic cycle, we have

$$
F+\mathrm{A}^{-}=V+\mathrm{D}^{+} \text {. }
$$

Taking the difference between equations (14) and (11) yields

$$
\Delta F+\mathrm{A}^{-}=\Delta V+\mathrm{D}^{+}
$$

where $\Delta F$ and $\Delta V$ denote the difference in concentration of color centers caused by surface chemical reactions compared to those in vacuo when only the physical (internal) pathway of excitation decay takes place. Consequently, equation (15) can be expressed 
differently as

$$
r_{\mathrm{ox}}+d[V] / d t=r_{\mathrm{red}}+d[F] / d t
$$

where $r_{\text {ox }}$ and $r_{\text {red }}$ are the rates of surface oxidation and reduction reactions, respectively, and $d[V] / d t$ and $d[F] / d t$ denote the rates of hole and electron trapping by defects. Equation (16) reflects the competition between internal and external cycles of the relaxation in heterogeneous systems. Our conclusions from equations (11) to (16) can be illustrated by our recent experimental results [1, 2] summarized in Figures 3 and 4 for the processes of formation of color centers during photoexcitation in the fundamental absorption band of solids in both solid/gas and solid/liquid heterogeneous systems.

Another illustration of the competition between chemical and physical pathways can be made on the basis of photobleaching of color centers. Absorption bands of photoinduced color centers formed during the photoexcitation of solids is typically red-shifted for up to a few eV compared to the intrinsic or extrinsic absorption bands. Photoexcitation can lead to photoionization of the color centers and consequently to generation of free charge carriers that can participate both in physical and chemical processes. As a result, the spectral limit of photochemical and photophysical processes is red-shifted in pre-photocolored solids compared to their initial states. Thus, photocoloration leads to the spectral sensitization of solids in heterogeneous systems [1, 2, 4, 6].

\section{PHOTOEXCITATION OF COLOR CENTERS}

For the case of photoexcitation of color centers, the probability of photoionization of these centers is either comparable to or greater than the probability of recombination decay. The fate of free charge carriers generated from the photoionization of color centers is the same as in previous considered cases as described by equations (3)-(5). Analysis of the kinetics and the mechanism of photobleaching yields for electron color centers:

$$
\begin{aligned}
& \left(\frac{d[F]}{d t}\right)_{A}-\left(\frac{d[F]}{d t}\right)_{\mathrm{vac}}=-\frac{d\left[A^{-}\right]}{d t}, \\
& \left(\frac{d[F]}{d t}\right)_{\mathrm{vac}}-\left(\frac{d[F]}{d t}\right)_{D}=-\frac{d\left[D^{+}\right]}{d t}
\end{aligned}
$$

and similarly for hole color centers:

$$
\begin{aligned}
& \left(\frac{d[V]}{d t}\right)_{\mathrm{vac}}-\left(\frac{d[V]}{d t}\right)_{A}=-\frac{d\left[A^{-}\right]}{d t}, \\
& \left(\frac{d[V]}{d t}\right)_{D}-\left(\frac{d[V]}{d t}\right)_{\mathrm{vac}}=-\frac{d\left[D^{+}\right]}{d t}
\end{aligned}
$$

which correspond to equation (16) and describe the experimental kinetics of photobleaching illustrated in

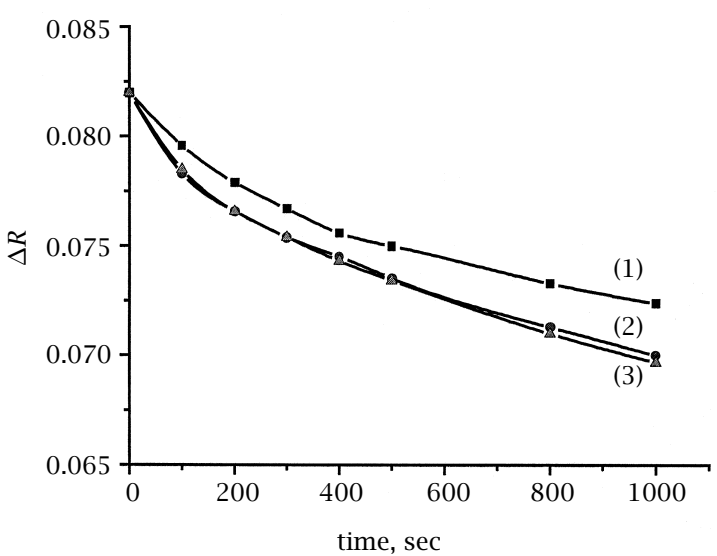

Figure 8. Kinetics of the photobleaching of $V$-type color centers in $\mathrm{Sc}_{2} \mathrm{O}_{3}$ in vacuo (1), in the presence of dihydrogen (2), and in the presence of $\mathrm{CO}$ (3). From reference [2] \{Copyright by the American Chemical Society?.

Figure 8. On the basis of the experimental data and the relationships between coloration and surface chemical processes described by equations (17) to (20), we were able to estimate the quantum yields of photobleaching processes together with the quantum yields of surface photochemical reactions for solid/gas heterogeneous systems involving $\mathrm{ZrO}_{2}$ and $\mathrm{Sc}_{2} \mathrm{O}_{3}$ specimens. Experimental quantum yields of photobleaching of $F$ centers in $\mathrm{ZrO}_{2}$ are (in vacuo) $\Phi_{F(\mathrm{vac})}=0.32 \pm 0.03$; in the presence of oxygen in the gas phase $\Phi_{F(\mathrm{O} 2)}=0.52 \pm 0.04$; and in the presence of dihydrogen $\Phi_{F(\mathrm{H} 2)}=0.22 \pm 0.03$. The quantum yields of photostimulated adsorption of dioxygen and dihydrogen during the photoexcitation of color centers are $\Phi_{\mathrm{O} 2}=0.16 \pm 0.02$ and $\Phi_{\mathrm{H} 2}=$ $0.11 \pm 0.02$, respectively. Clearly, the relationship between measured quantum yields considering dissociative adsorption for dihydrogen on surface hole centers can be presented in the form,

$$
\begin{gathered}
\Phi_{F(\mathrm{O} 2)}-\Phi_{F(\mathrm{vac})}=\Phi_{\mathrm{O} 2}, \\
\Phi_{F(\mathrm{vac})}-\Phi_{F(\mathrm{H} 2)}=2 \Phi_{\mathrm{H} 2}
\end{gathered}
$$

in complete accord with equations (17) and (18).

The same is true for the heterogeneous system involving scandia $\mathrm{Sc}_{2} \mathrm{O}_{3}$ photoexcited in the absorption band of $V$-type hole color centers. The quantum yield of photobleaching of $V$-type color centers was $\Phi_{V}=$ $0.022 \pm 0.002$ in vacuo, and $\Phi_{V}=0.046 \pm 0.003$ in a dihydrogen or carbon monoxide atmosphere. The quantum yield of the photostimulated adsorption of dihydrogen and carbon monoxide on colored scandia is $\Phi=0.023 \pm 0.002$, so that the quantum yield of photobleaching of hole color centers in dihydrogen (or carbon monoxide) reflects the sum of the quantum yield of photobleaching of coloration in vacuo and the quantum 
yield of surface photochemical processes. That is,

$$
\Phi_{V(\mathrm{H} 2)}-\Phi_{V(\mathrm{vac})}=\Phi_{\mathrm{H} 2} .
$$

Ionization of photoinduced color centers can also be caused by an increase in temperature, which increases the probability of the thermostimulated decay of color centers, $q_{\text {th }}$ (see equation (1)). Thermoionization and photoionization of color centers generate free charge carriers. These can participate in both physical and chemical processes. Examples of such processes and their correlations are presented in Figure (9). Figure 9a depicts the temperature dependencies of the decay of $V$-type hole color centers in $\mathrm{Sc}_{2} \mathrm{O}_{3}$ and of the extent of hydrogen adsorption. The latter is not observed in non-colored samples [6]. The dependencies are caused by the appearance of holes on the surface generated
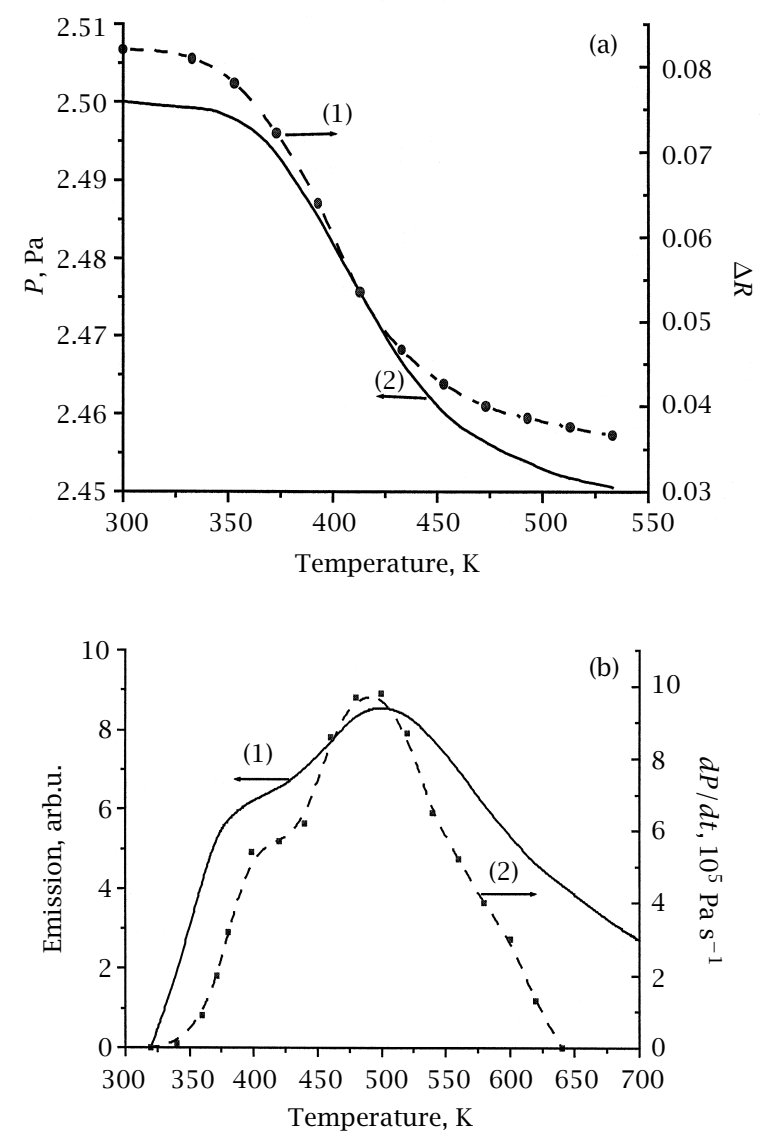

Figure 9. (a) Temperature dependence of the absorption of $V$-type color centers in $\mathrm{Sc}_{2} \mathrm{O}_{3}(1)$, and the thermostimulated adsorption of dihydrogen (2). From reference [2] \{Copyright by the American Chemical Society\}. (b) Temperature dependencies of the thermostimulated emission (1) and of the rate of the thermostimulated adsorption of dihydrogen on $\mathrm{MgAl}_{2} \mathrm{O}_{4}$. From reference [6] \{Copyright by the American Chemical Society\}. from the thermal ionization of hole color centers (equations (24), (25)).

$$
\begin{gathered}
V \longrightarrow \mathrm{V}_{\mathrm{c}}+\mathrm{h}, \\
\mathrm{h}\left(\mathrm{O}_{\mathrm{s}}{ }^{-\bullet}\right)+\mathrm{H}_{2} \longrightarrow \mathrm{OH}_{\mathrm{s}}{ }^{-}+\mathrm{H} \bullet
\end{gathered}
$$

Similar effects have been observed for the heterogeneous systems $\mathrm{MgAl}_{2} \mathrm{O}_{4} / \mathrm{H}_{2}$ and $\mathrm{ZrO}_{2} / \mathrm{O}_{2}$ where a correlation between the rate of thermostimulated adsorption and the intensity of thermostimulated luminescence was established (Figure 9b) $[4,6,16]$. In this case, thermostimulated luminescence was caused by free charge carrier trapping that accompanied emission of photons:

$$
\mathrm{h}+\mathrm{V}_{\mathrm{c}} \rightarrow V+\mathrm{h} v
$$

Again this physical process (26) competes with the chemical process of reaction (25). In the $\mathrm{ZrO}_{2} / \mathrm{O}_{2}$ heterogeneous system, the thermal destruction of electron color centers is responsible for the appearance of free electrons participating in both thermoluminescence and oxygen adsorption processes.

It is worth summarizing all the processes considered above. They are displayed in some detail in Scheme 1 .

\section{Bulk processes}

1. Intrinsic photoexcitation of the bulk lattice $L_{b}$ (fundamental absorption of light with photon energy $\left.h v_{1} \gtrsim E_{g}\right)$ leads to generation of free electrons $\left(e^{-}\right)$and holes $\left(h^{+}\right)$, or excitons $\left(e^{o}\right)($ step 1$)$.

2. Extrinsic photoexcitation of pre-existing bulk defects $D_{b}$ by light with photons energy $h v_{2}<h v_{1}$ also leads to generation of free electrons and holes (step 8).

3. Trapping of free charge carriers and excitons by the bulk defects form color ( $F$ - and $V$-type) centers (step 2) in the bulk.

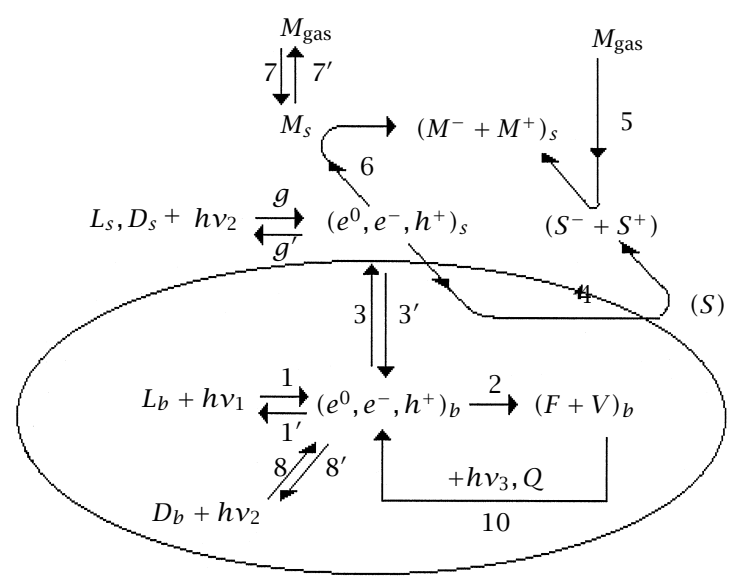

Scheme 1. Summary of photostimulated processes in a heterogeneous system containing a wide bandgap solid. 
4. Photobleaching $\left(h v_{3}\right)$ and thermo-annealing $(Q)$ of color centers leads to generation of free electrons and holes (step 10).

5. Band-to-band recombination of free charge carriers and exciton decay (step 1'), and recombination and exciton decay through defects (step 8') is likely accompanied by luminescence ( $h v_{1}$ and $h v_{2}$, respectively).

6. Free carrier and exciton exchange between the bulk and the surface due to diffusion and/or drift of these carriers (steps 3 and 3').

\section{Surface processes}

1. Light absorption $\left(h v_{2}\right)$ by regular and irregular surface states $L_{s}$ and $D_{s}$ leads to generation of surface free charge carriers and surface excitons (step 9).

2. Charge carrier and exciton trapping by preexisting surface defects $(S)$ yields surface active centers $\left(S^{-}\right.$and $\left.S^{+}\right)$for adsorption and catalysis (step 4).

3. Interaction of molecules in the gas (or liquid) phase, $\mathrm{M}_{\mathrm{gas}}$, with surface active centers initiates surface chemical processes (step 5; the EleyRideal mechanism).

4. Adsorption and desorption of molecules in the gas (or liquid) phase (steps 7 and 7') lead to adsorption/desorption equilibria.

5. Interaction of surface charge carriers and excitons with pre-adsorbed molecules $\left(\mathrm{M}_{\mathrm{S}}\right)$ initiates surface chemical processes (step 6; the Langmuir-Hinshelwood mechanism).

6. Surface recombination of free charge carriers and surface exciton decay (step 9') occurs and is likely accompanied by luminescence.

\section{THEORETICAL DESCRIPTION OF QUANTUM YIELDS IN HETEROGENEOUS SYSTEMS}

It is evident in Scheme 1 that there always exists competition between different photophysical and photochemical processes. The efficiency of each process is determined by the efficiencies of others. The efficiency of surface photochemical reactions is typically characterized by the quantum yield of the photoreaction occurring on the particle surface. The quantum yield in heterogeneous media has been described $[17,18]$ in a manner identical to that in homogeneous photochemistry which defines it (equation (27)) as the ratio of the number of molecules that undergo a chemical transformation per unit time, $d N_{r} / d t$ (molecules $\mathrm{s}^{-1}$ ), to the number of photons absorbed by the heterogeneous system per unit time at a given wavelength, $d N_{h v}(a b s) / d t$ (photons s ${ }^{-1}$ ), equation (27).

$$
\Phi=\frac{d N_{r} / d t}{d N_{h v(a b s)} / d t} .
$$

Experimental measurements of the reaction rate create no problems and can be done by simple kinetic measurements of the (initial) reaction rate. However, appropriate determination of the fraction of absorbed light in heterogeneous systems has made the measurements of quantum yields a complex issue. This problem was solved for solid/gas heterogeneous systems using either diffuse reflectance spectroscopy with a standard reference sample, or a black body-like reactor [2$4,6,14,19]$. The experimental protocol for determining the fraction of absorbed light in solid/liquid heterogeneous systems was reported recently by Serpone and coworkers $[17,18]$ using an integrating sphere assembly.

As noted earlier, the rate of a surface photochemical reaction depends on the concentration of the reagent and on photon flow (see equation (9)). In the general case then the quantum yield of the reaction will also depend on these two parameters. Indeed, substituting the reaction rate expression (equation (9)) into expression (27) gives,

$$
\Phi(\rho,[M])=\frac{\alpha[M]}{A(\beta \rho+\gamma[M])},
$$

where $A$ represents the fraction of the photon flow absorbed by the heterogeneous system. It is rather obvious that the necessary condition to determine the highest value of the quantum yield that would be independent of the concentration and photon flow occurs when $\gamma[M] \gg \beta \rho$. Note that this condition corresponds to the highest efficiency of the chemical reaction in competition with recombination through the surface active centers, when recombination is completely shut down and the reaction rate is determined solely by the rate of generation of surface active sites. Note also that under such conditions, to achieve the higher efficiency for a given photocatalyst at lower concentrations of reagent requires a lower photon flow.

To gain a better understanding of those factors that determine the efficiency (quantum yield) of heterogeneous photocatalytic processes we examined a theoretical approach that required consideration of both bulk and surface processes in the photocatalyst, along with primary and secondary chemical surface reactions [20]. At sufficiently high concentrations of reagent, when the chemical reaction dominates the physical pathway of surface recombination of carriers, the reaction rate is equal to the rate of generation of surface active centers which in turn is proportional to the surface concentration of free charge carriers of the corresponding sign 
(see stages (5a)-(5c)).

$$
\left(\frac{d[M]}{d t}\right)=k_{e}\left[e_{s}\right][S] .
$$

The concentration of surface charge carriers can be found from the solution to the continuity equation under the steady-state approximation:

$$
\frac{\partial n(x, t)}{\partial t}=-\frac{\partial J_{n}(x, t)}{\partial x}-\frac{n(x, t)}{\tau}+G(x, t)=0
$$

with the given boundary conditions given by,

$$
J_{s}=s n_{s},
$$

where $n(x, t)$ is the concentration of free charge carriers at a given point in space with spatial coordinates $x$ at a given time $t ; J(x, t)$ is the flow of charge carriers caused either by diffusion or by drift; $G(x, t)$ is a timespace function of carrier photogeneration; $\tau$ is the lifetime of carriers in the bulk of the solid; and $s$ is the rate constant of surface carrier recombination. The solution to the continuity equation (30) for a one-dimensional infinite plate model taking into consideration a nonuniform function of carrier generation that obeys the Lambert-Beer law and with diffusion as the major path of carrier flow gives the spatial distribution of carriers in the bulk of the solid (see Figure 10). The expression for the surface concentration of carriers is then given by [20],

$$
\begin{aligned}
n_{s}= & \frac{2\left(1-e^{-\alpha d}\right) \chi \rho \alpha L^{2}}{D(\tanh (d / 2 L)+\xi)\left(1-\alpha^{2} L^{2}\right)} \\
& \times\left[\tanh \left(\frac{d}{2 L}\right) \operatorname{coth}\left(\frac{\alpha d}{2}\right)-\alpha L\right] .
\end{aligned}
$$

In equation (32), $\alpha$ is the absorption coefficient of the solid, $d$ is the thickness of the plate, $L=(D \tau)^{1 / 2}$ is the diffusion length of the charge carriers, and $\xi$ is a ratio of the rates of surface to bulk recombinations. Substitution of the surface concentration of carriers (equation (32)) into equation (29) for the reaction rate, followed by substitution of the resulting expression into equation (27) for the quantum yield \{considering that the fraction of absorbed photons is $A=2\left(1-e^{-\alpha d}\right)$ for the infinite plate irradiated from both sides\} results in the expression for the quantum yield $\Phi$ of the primary surface chemical process given by equation (33):

$$
\begin{aligned}
\Phi= & \frac{k_{e} S \chi \alpha L^{2}}{D(\tanh (d / 2 L)+\xi)\left(1-\alpha^{2} L^{2}\right)} \\
& \times\left[\tanh \left(\frac{d}{2 L}\right) \operatorname{coth}\left(\frac{\alpha d}{2}\right)-\alpha L\right] .
\end{aligned}
$$

Several inferences can be made from this last expression for the quantum yield. The greater the probability of the chemical pathway, which is proportional to $k_{e} S$, the greater is the quantum yield of the photochemical reaction. However the efficiency of the physical pathway also plays a key role. The greater the efficiencies of the physical pathways the smaller is the quantum yield of the photoreaction. Indeed, a faster rate of surface recombination increases the value $\xi$ in the denominator, whereas a faster rate of bulk recombination decreases the lifetime of the charge carriers, $\tau$, and so decreases the diffusion length $L$ in the numerator from the relationship $L=(D \tau)^{1 / 2}$.

Equation (33) also infers that a spectral variation of the absorption coefficient $\alpha$ leads to a spectral variation of the quantum yield of chemical processes, and permits inferences on the conditions which may determine whether the quantum yield is spectrally dependent or spectrally independent. Specifically, the quantum yield becomes spectrally independent in the case of very strong fundamental absorption (i.e. when $\alpha \rightarrow$ $\infty)$, and when absorption of the incident light is weak (i.e. for $\alpha \rightarrow 0$ ) corresponding to extrinsic light absorption (see Figure 11). The quantum yield is also spectrally independent when the surface states absorb the incident radiation. When $\alpha L$ is near unity, $\Phi$ becomes spectrally variable and increases with increasing absorption coefficient (see e.g. Figure 12). The reasons for such a behavior are that the spectral variations of the absorption coefficient change the number

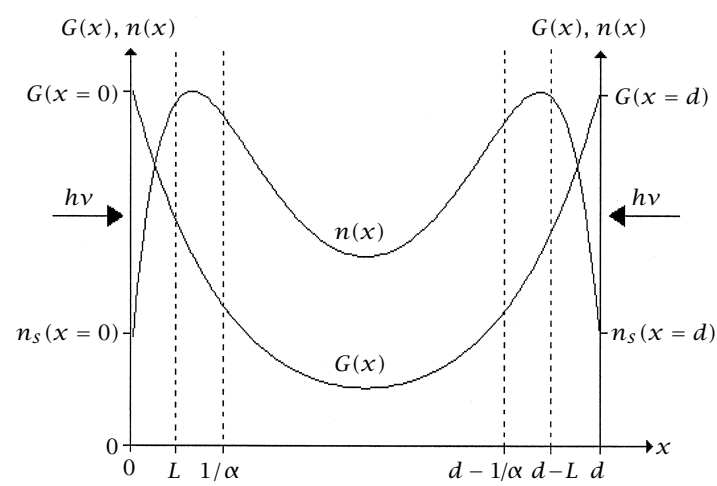

Figure 10. Scheme of an infinite solid plate of thickness $d$ irradiated uniformely from both sides. Curve $G(x)$ denotes a nonuniform spatial distribution of carrier photogeneration. Straight vertical lines between the plate surface and the bulk represent the depth equal to the average diffusion length of the carriers $L$ and average length $1 / \alpha$ of light penetration into the bulk. Carriers generated within the diffusion length can reach the plate surfaces by diffusive migration. Carriers generated in the rest of the lattice bulk do not take part in surface chemical processes. Curve $n(x)$ represents the spatial distribution of the concentration of free charge carriers. From reference [20] \{Copyright by the American Chemical Society\}. 


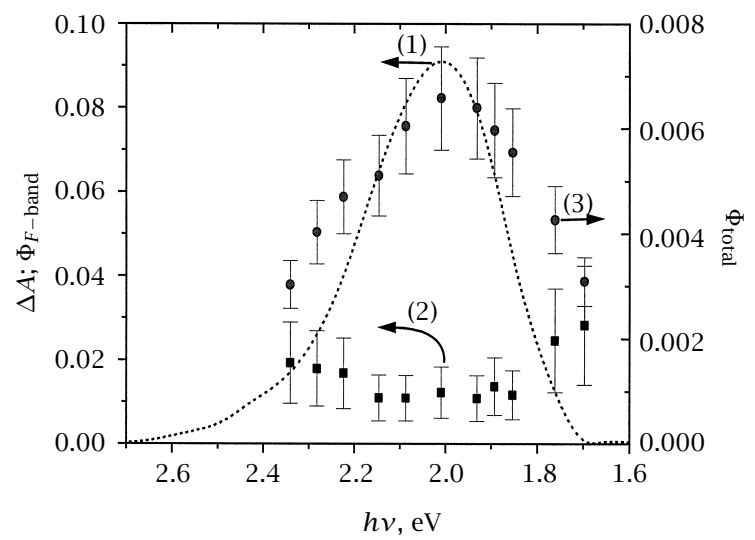

Figure 11. Absorption spectrum of $F$ color centers in $\mathrm{KBr}$ (1), and spectral dependence of the quantum yield of photoadsorption of molecular oxygen considering only the absorption of F centers (2) and the total (active and inactive) absorption of $\mathrm{KBr}$ (3). From reference [3] \{Copyright by the American Chemical Society?.

of charge carriers generated within the diffusion length $(L)$ able to reach the surface and to participate in surface photoreactions. For weak light absorption, the ratio of the number of such carriers to the number of absorbed photons is a constant, so that the quantum yield of a surface photoreaction for a given photocatalyst depends on the ratio $d / 2 L$, which in the one-dimensional model is proportional to the ratio between the total volume of the bulk crystal $\left(\mathrm{V}_{\text {crys. }} \propto d\right)$ and the volume of the bulk space near the surface $\left(\mathrm{V}_{\text {diff }} \propto 2 L\right)$ from which photogenerated carriers can reach the surface by diffusion. In the spectral region where the absorption coefficient increases, more carriers are generated within the diffusion length leading to an increase in the quantum yield of surface chemical reactions. For the case of very strong absorption, all the carriers are generated within the diffusion length with a certain probability of participating in surface processes.

More complex events take place when the absorption spectra of the solids are formed by the overlap of several single absorption bands that belong to different types of light absorption, and that differ either (i) by their ability to form free carriers (internal quantum yield of photoeffect, $\chi$ ) and/or (ii) by the properties (e.g., mobility, lifetime) of newly generated carriers. In this case, the total quantum yield of the surface photochemical reaction is given by,

$$
\Phi=\frac{R}{A \rho}=\frac{\sum_{i} A_{i} \Phi_{i}}{\sum_{i} A_{i}},
$$

where $R=\sum R_{i}$ and $R_{i}\left\{=A_{i} \rho \phi_{i}\right\}$ is the rate of the elementary reaction under excitation in the $i$-th single absorption band, $A_{i}$ is the absorbance in the $i$-th single absorption band, and $\Phi_{i}$ is the corresponding quantum yield of the reaction. The total number of absorbed photons per unit time is given by $A \rho=\rho \sum_{i} A_{i}$ (where $A$ is the total absorbance at a given wavelength).

We have demonstrated both theoretically and experimentally [3, 20, 21] that the former reason (i) is essential for weak extrinsic light absorption by defects, which leads to band-like or step-like spectral dependencies of the quantum yields (see Figures 11 and 13) whose shape is dictated by the degree of overlap of the single absorption bands of different types (particularly by the degree of overlap of active and inactive light absorption), whereas the latter reason (ii) becomes important in the spectral range of intrinsic (fundamental) light absorption by the solid when the hot carriers generated at a photon energy greater than the bandgap are involved in surface processes. In such a case, in addition to the spectral variations of $\Phi$ caused by the spectral variation of the absorption coefficient $\alpha$ (see above), especially at the fundamental absorption edge, the spectral dependence of $\Phi$ may also be attributed to the spectral variation of the mobilities and lifetimes of the charge carriers generated at different wavelengths (see equation (33)) owing to different direct and indirect band-to-band transitions that result in different initial population of the electronic states in the valence and conduction bands. This latter factor becomes important when communication between different subbands (in the Brillouin zone) is weak enough to render fast thermalization of hot carriers unlikely. In such a case, competition between diffusion of carriers toward the surface and their energy losses is quite effective in

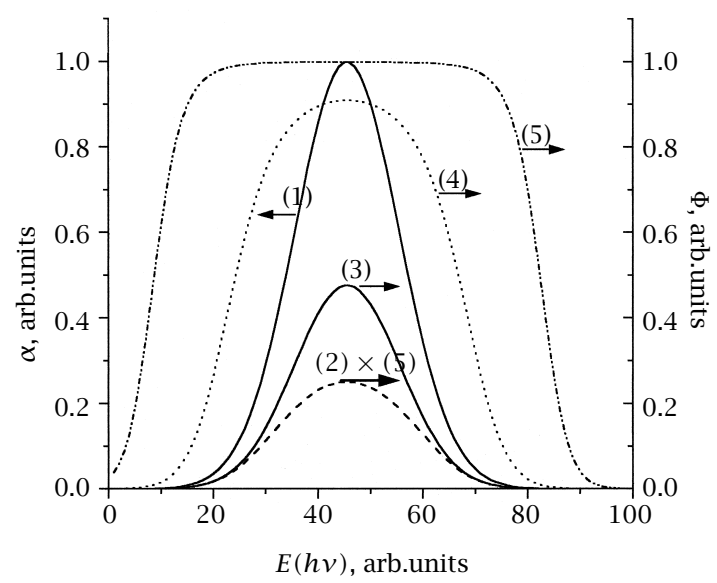

Figure 12. Spectral dependence of the quantum yield within a single absorption band (curve (1)) for different maximal values of the co-relationship $\alpha L$ : curve (2), $\alpha L=0.1$; curve (3), $\alpha L=1$; curve (4), $\alpha L=10$; and curve (5), $\alpha L=1000$. From reference [20] \{Copyright by the American Chemical Society\}. 
small particles; this promotes the involvement of carriers with excess energy into surface processes. The latter may also affect the reactivity of the surface active sites.
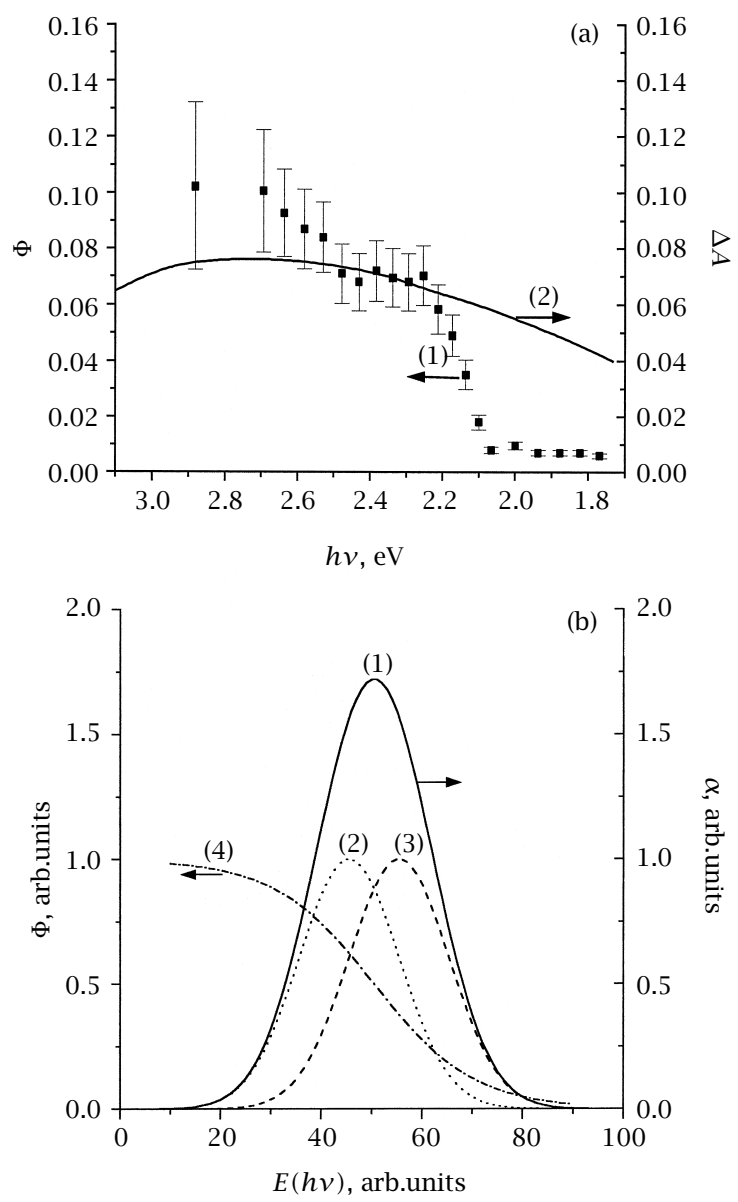

Figure 13. (a) Step-like spectral dependence of the quantum yield of the photostimulated adsorption of dioxygen on colored $\mathrm{ZrO}_{2}$ particles (1), and absorption spectrum of $F$ type color centers (2). From reference [3] \{Copyright by the American Chemical Society\}. (b) Theoretical step-like spectral dependence of the quantum yield (4) for the case of a complex absorption band (1) formed by the overlap of two single absorption bands $(2,3)$ of two different types of defects.

Experimental spectral dependencies of $\Phi[3,21]$ of surface photochemical processes in illuminated aqueous $\mathrm{TiO}_{2}$ dispersions (Figures 14) both in rutile and in anatase forms demonstrate a band-like structure with maxima corresponding to the energies of indirect and direct transitions in $\mathrm{TiO}_{2}$. This infers that photocarriers generated at different wavelengths of excitation do differ in their mobilities and activities both in the bulk and in the surface processes.
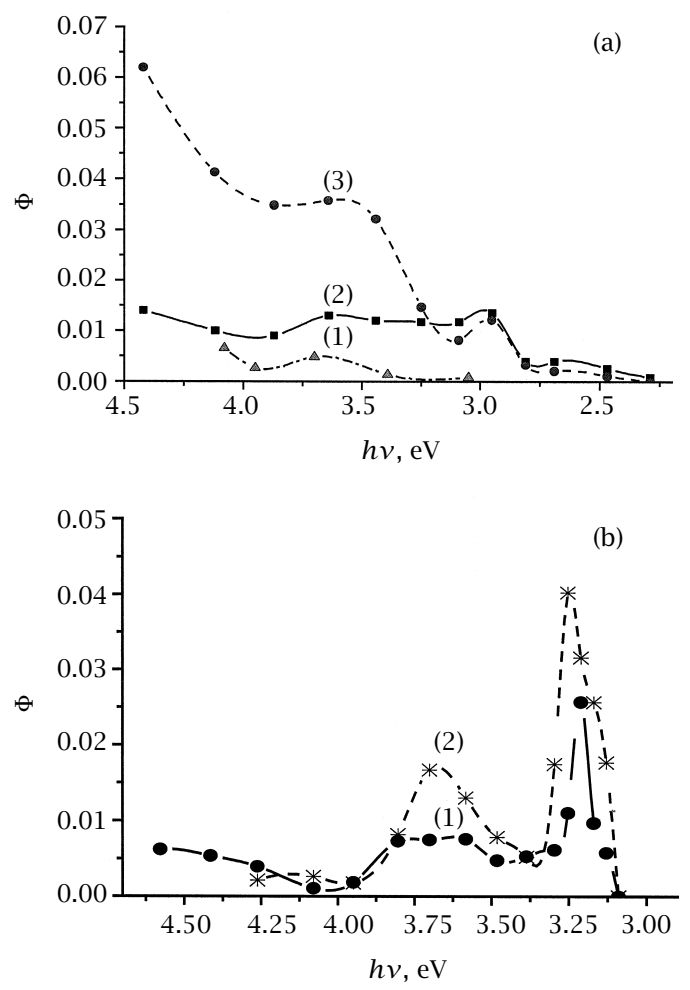

Figure 14. (a) Spectral dependencies of the quantum yields of the photostimulated adsorption of dioxygen (1), dihydrogen (2), and methane (3) on powdered $\mathrm{TiO}_{2}$ (rutile). From reference [3] \{Copyright by the American Chemical Society?. (b) Spectral dependencies of the quantum yields of the photodegradation of phenol (1), and 4-chlorophenol (2) in illuminated aqueous $\mathrm{TiO}_{2}$ dispersions (Degussa P-25 titania). From reference [21] \{Copyright by the American Chemical Society?.

\section{CONCLUDING REMARKS}

While much has been done to gain a fairly good appreciation of heterogeneous photocatalysis, this article has shown that absorption of incident light by a metal oxide photocatalyst leads to a very complex series of photophysical and photochemical events in the bulk, in the space charge layer, and on the surface of the metal oxide particles. The most dramatic discovery was that the quantum yield, for which a protocol is now available for its assessment in heterogeneous media, of photoredox reactions, that of necessity take place at the particle surface, is wavelength dependent. This aspect has a number of implications. For example, the mobilities and lifetimes of the charge carriers depend on the wavelength of irradiation, but more importantly hot carriers formed at short UV wavelengths of excitation do not relax as rapidly in their respective band as conventional wisdom has dictated for some time. We have also in- 
ferred that, in this event, it is not unlikely for the primary oxidizing entity on the metal-oxide surfaces be the surface-bound $\bullet \mathrm{OH}$ radicals in some excited state. Of necessity, such an inference requires further work from both a theoretical and experimental viewpoint to lay down the foundations for a more comprehensive understanding of what actually takes place on the surface subsequent to absorption of photons by metal-oxide particles of semiconductors and dielectrics.

\section{ACKNOWLEDGEMENTS}

We are grateful to the Natural Science and Engineering Research Council of Canada for support of our work.

\section{REFERENCES}

[1] A. V. Emeline, G. V. Kataeva, A. S. Litke, A. V. Rudakova, V. K. Ryabchuk, and N. Serpone, Langmuir 14 (1998), 5011.

[2] A. V. Emeline, S. V. Petrova, V. K. Ryabchuk, and N. Serpone, Chem. Mater. 10 (1998), 3484.

[3] A. V. Emeline, G. N. Kuzmin, D. Purevdorj, V. K. Ryabchuk, and N. Serpone, J. Phys. Chem. B 104 (2000), 2989.

[4] V. K. Ryabchuk and G. V. Burukina, Sov. J. Phys. Chem. 65 (1991), 1621.

[5] A. R. Siline and A. N. Trukchin, Point Defects and Elementary Excitation in Crystalline and Glass $\mathrm{SiO}_{2}$, Zinatne Publishing House, Riga, 1985.

[6] A. V. Emeline, G. V. Kataeva, V. K. Ryabchuk, and N. Serpone, J. Phys. Chem. B. 103 (1999), 9190.

[7] M. R. V. Sahyun and N. Serpone, Langmuir 13 (1997), 5082.
[8] V. G. Baru and Th. Th. Volkenstein, The Effect of Irradiation on Surface Properties of Semiconductors, Nauka, Moscow, Russia, 1978.

[9] G. Lu, A. Linsebigler, and J. T. Yates Jr., J. Chem. Phys. 102 (1995), 4657.

[10] A. Linsebigler, G. Lu, and J. T. Yates Jr., Chem. Rev. 95 (1995), 735.

[11] M. Formenti and S. I. Teichner, J. Catal. 2 (1978), 87.

[12] A. V. Emeline, V. K. Ryabchuk, and N. Serpone, J. Photochem. Photobiol. A-Chemistry 133 (2000), 89.

[13] A. V. Emeline, A. V. Rudakova, V. K. Ryabchuk, and N. Serpone, J. Phys. Chem. B 102 (1998), 10906.

[14] L. L. Basov, G. N. Kuzmin, I. M. Prudnikov, and Yu. P. Solonitzyn, Uspekhi Photoniki., Th. I. Vilesov (ed.), LGU (Leningrad State University), 6 (1977), 82.

[15] D. F. Ollis, Photochemical Conversion and Storage of Solar Energy, E. Pelizzeti and M. Schiavello (eds.), Kluwer, Dordrecht, 1991, pp. 593-622.

[16] A. V. Emeline and V. K. Ryabchuk, Russ. J. Phys. Chem. 72 (1998), 432.

[17] A. Salinaro, A. V. Emeline, J. Zhao, H. Hidaka, V. K. Ryabchuck, and N. Serpone, Pure \& Appl. Chem. 71 (1999), 321.

[18] N. Serpone and A. Salinaro, Pure \& Appl. Chem. 71 (1999) 303.

[19] V. N. Kuznetsov and A. A. Lisachenko, Sov. J. Phys. Chem. 65 (1991), 1568.

[20] A. V. Emeline, V. K. Ryabchuk, and N. Serpone, J. Phys. Chem. B. 103 (1999), 1316.

[21] A. V. Emeline, A. Salinaro, and N. Serpone, J. Phys. Chem. B 104 (2000), 11202. 


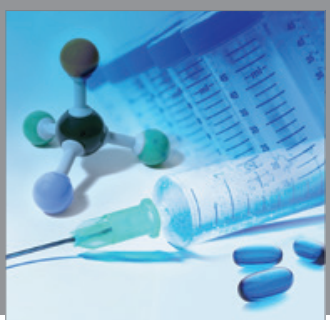

International Journal of

Medicinal Chemistry

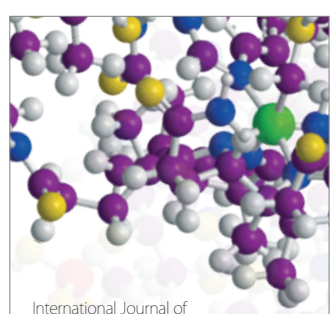

Carbohydrate Chemistry

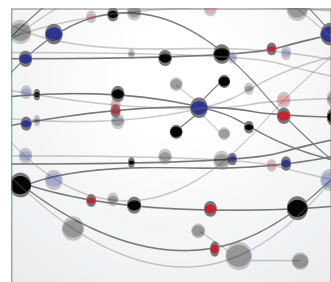

The Scientific World Journal
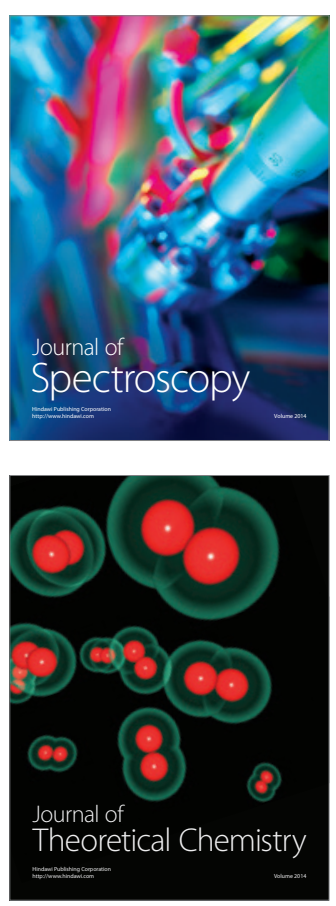
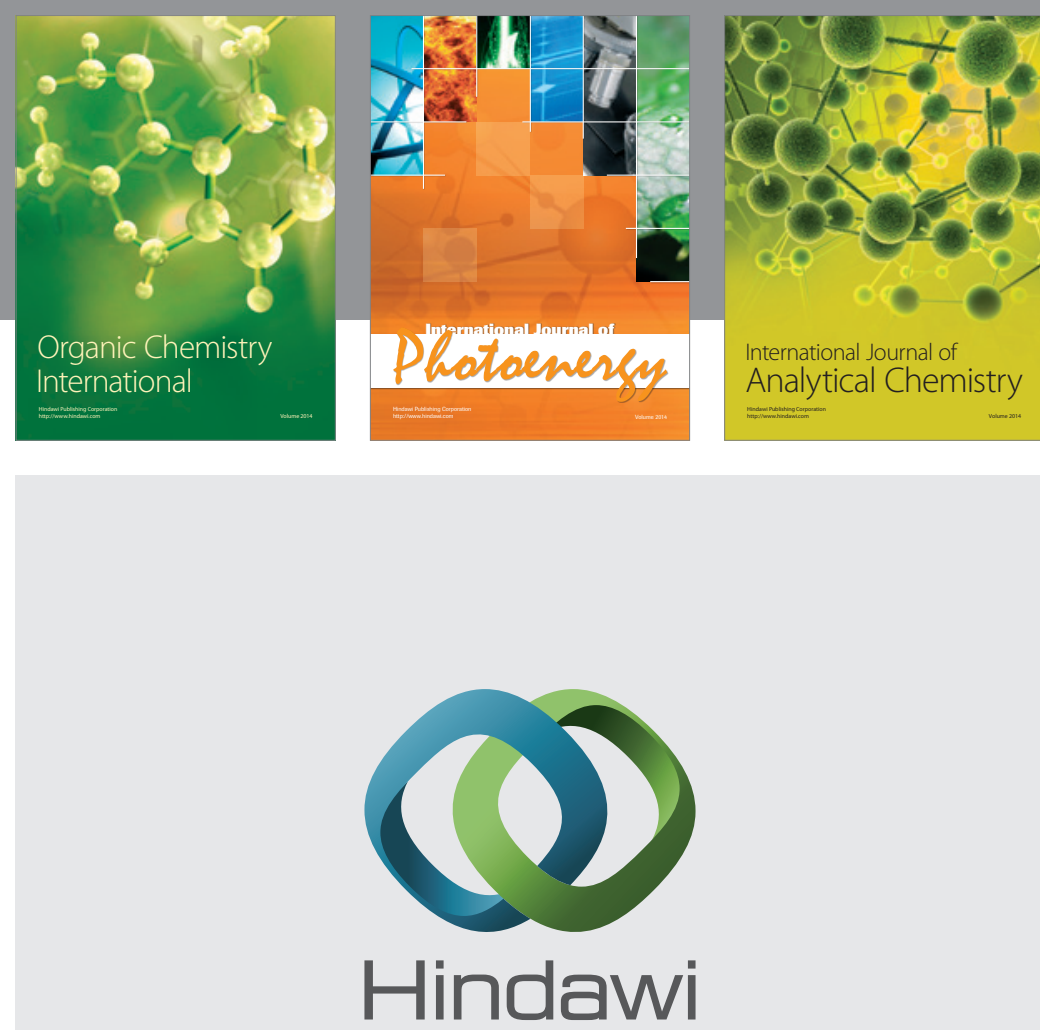

Submit your manuscripts at

http://www.hindawi.com
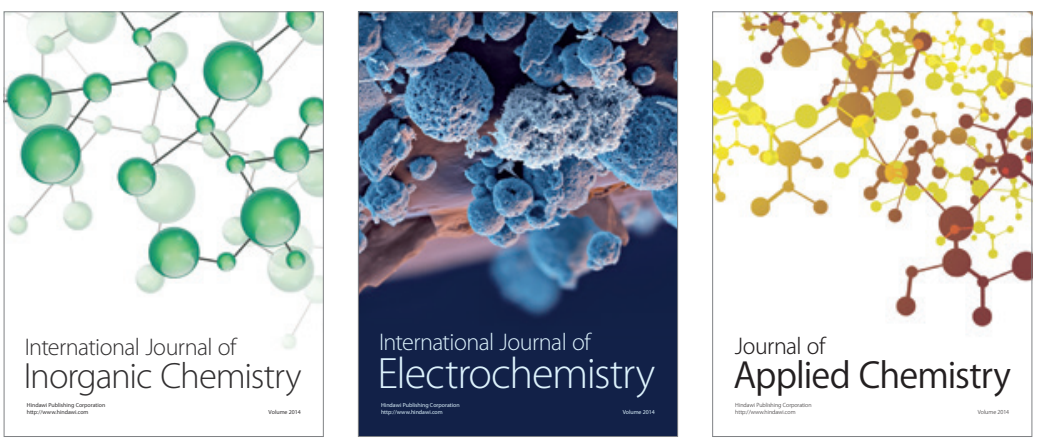

Journal of

Applied Chemistry
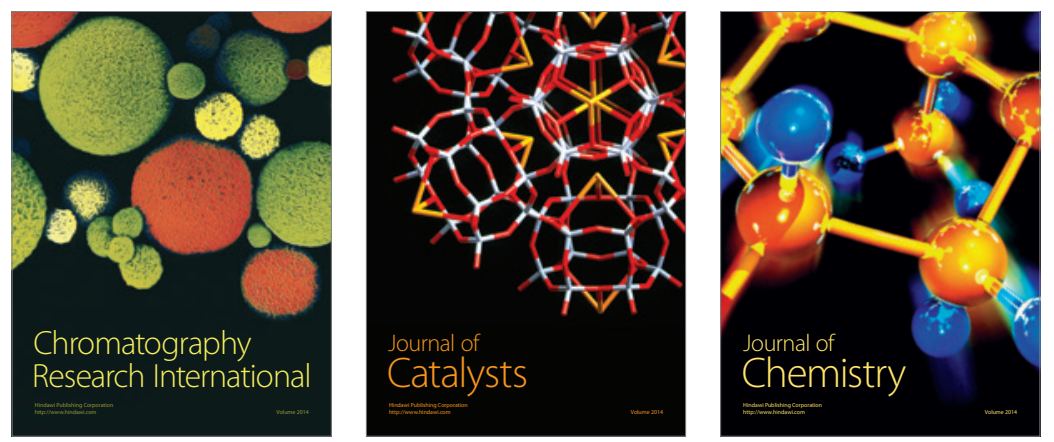
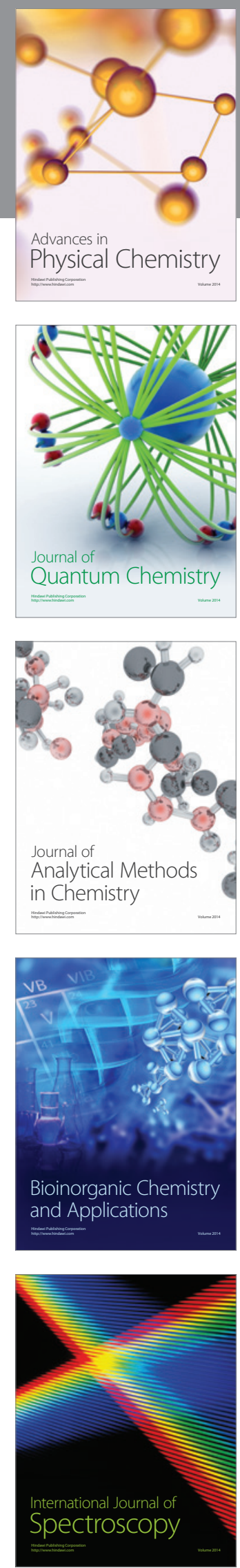